
Physics

\title{
Some compatible Poisson structures and integrable bi-Hamiltonian systems on four dimensional and nilpotent six dimensional symplectic real Lie groups
}

Jafar Abedi-Fardad, Adel Rezaei-Aghdam, Ghorbanali Haghighatdoost

To cite this article: Jafar Abedi-Fardad, Adel Rezaei-Aghdam, Ghorbanali Haghighatdoost (2017) Some compatible Poisson structures and integrable bi-Hamiltonian systems on four dimensional and nilpotent six dimensional symplectic real Lie groups, Journal of Nonlinear Mathematical Physics 24:2, 149-170, DOI:

https://doi.org/10.1080/14029251.2017.1306944

To link to this article: https://doi.org/10.1080/14029251.2017.1306944

Published online: 04 January 2021 


\title{
Some compatible Poisson structures and integrable bi-Hamiltonian systems on four dimensional and nilpotent six dimensional symplectic real Lie groups
}

\author{
Jafar Abedi-Fardad \\ Department of Mathematics, University of Bonab, Tabriz, Iran \\ j.abedifardad@bonabu.ac.ir \\ Adel Rezaei-Aghdam \\ Department of Physics, Azarbaijan Shahid Madani University, 53714-161, Tabriz, Iran \\ rezaei-a@azaruniv.edu \\ Ghorbanali Haghighatdoost \\ Department of Mathematics, Azarbaijan Shahid Madani University, 53714-161, Tabriz, Iran \\ gorbanali@azaruniv.edu
}

Received 22 October 2016

Accepted 18 January 2017

\begin{abstract}
We provide an alternative method for obtaining of compatible Poisson structures on Lie groups by means of the adjoint representations of Lie algebras. In this way we calculate some compatible Poisson structures on four dimensional and nilpotent six dimensional symplectic real Lie groups. Then using Magri-Morosi's theorem we obtain new bi-Hamiltonian systems with four dimensional and nilpotent six dimensional symplectic real Lie groups as phase spaces.
\end{abstract}

Keywords: Integrable bi-Hamiltonian system; Compatible Poisson structures; Symplectic Lie group.

\section{Introduction}

Compatible different Hamiltonian structures have been proved to be a valuable tool in the construction of infinite hierarchies of symmetries and conservation laws for PDEs (see e.g. KdV equation [5]). Kdv equation can be studied as a bi-Hamiltonian system. The study of bi-Hamiltonian systems started with the pioneering work of Magri [10] and developed later in many papers (see for example [8], [9] and [2] ). The bi-Hamiltonian structure has been observed in many of classical systems and some new interesting examples of bi-Hamiltonian systems have been discovered (see for example [20], [6] and [21]). In this work, we give a method to construct compatible Poisson structures on a Lie group by means of the adjoint representation of its Lie algebra and construct integrable bi-Hamiltonian systems by using Magri-Morosi's theorem [12] (for a review see [11]). Of course using of adjoint representation in the context of the coadjoint orbit method applied previously in the Hamiltonian system (see for example [11] and [18]). We will give a method to produce integral of motion of a non-degenerate bi-Hamiltonian systems, for which the Lie group is the phase space.

The outline of the paper is as follows. In section two after reviewing the definition of compatible Poisson structures we give a method for calculating compatible Poisson structures on a Lie group (in general). Then in section three we have obtained these structures on symplectic four dimensional real Lie groups. In section four using Magri-Morosi's theorem we have obtained new 
bi-Hamiltonian systems on symplectic four dimensional real Lie groups (as phase space). In the same way we have obtained the compatible Poisson structures on symplectic nilpotent six dimensional real Lie groups [14] (see [19] for a rigorous commutative relations) in section five, of course in this section we have obtained vielbeins on symplectic nilpotent six dimensional real Lie algebras, where the results are summarized in appendix B. Finally in section six some new bi-Hamiltonian systems on symplectic nilpotent six dimensional real Lie groups have been obtained. Some concluding remarks are given in section seven. The list of four dimensional and nilpotent six dimensional symplectic real Lie algebras are given in appendix A and B.

\section{Compatible Poisson structures and bi-Hamiltonian systems on Lie groups}

\subsection{Definitions and Notations}

For the sake of completeness of the paper let us have a short review on the compatible Poisson structures and bi-Hamiltonian system (for a review see [11]).

Definition: [12] A pair of Poisson brackets $\{.,$.$\} and \{., .\}^{\prime}$ or a pair of Poisson bivectors $\mathbf{P}$ and $\mathbf{P}^{\prime}$ on an $m$ dimensional manifold $\mathbf{M}$ is called compatible if we have:

$$
[\mathbf{P}, \mathbf{P}]=\left[\mathbf{P}^{\prime}, \mathbf{P}^{\prime}\right]=\left[\mathbf{P}, \mathbf{P}^{\prime}\right]=0,
$$

where $[.,$.$] is the Schouten bracket that have the following forms:$

$$
\begin{gathered}
{[\mathbf{P}, \mathbf{P}]^{\lambda \mu v}=\mathbf{P}^{\rho \lambda} \partial_{\rho} \mathbf{P}^{\mu v}+\mathbf{P}^{\rho v} \partial_{\rho} \mathbf{P}^{\lambda \mu}+\mathbf{P}^{\rho \mu} \partial_{\rho} \mathbf{P}^{v \lambda}} \\
{\left[\mathbf{P}^{\prime}, \mathbf{P}^{\prime}\right]^{\lambda \mu v}=\mathbf{P}^{\prime \rho \lambda} \partial_{\rho} \mathbf{P}^{\prime \mu v}+\mathbf{P}^{\prime \rho v} \partial_{\rho} \mathbf{P}^{\prime \lambda \mu}+\mathbf{P}^{\prime \rho \mu} \partial_{\rho} \mathbf{P}^{\prime v \lambda}} \\
{\left[\mathbf{P}, \mathbf{P}^{\prime}\right]^{\lambda \mu v}=\mathbf{P}^{\rho \lambda} \partial_{\rho} \mathbf{P}^{\prime \mu v}+\mathbf{P}^{\prime \rho \lambda} \partial_{\rho} \mathbf{P}^{\mu v}+\mathbf{P}^{\rho v} \partial_{\rho} \mathbf{P}^{\prime \lambda \mu}+\mathbf{P}^{\prime \rho v} \partial_{\rho} \mathbf{P}^{\lambda \mu}+\mathbf{P}^{\rho \mu} \partial_{\rho} \mathbf{P}^{\prime v \lambda}+\mathbf{P}^{\prime \rho \mu} \partial_{\rho} \mathbf{P}^{v \lambda}}
\end{gathered}
$$

where $\partial_{\rho}=\frac{\partial}{\partial x_{\rho}}$ such that $\left(x_{1}, \ldots, x_{m}\right)$ is the coordinate of the manifold M. The Poisson bracket corresponding to the Poisson bivector $\mathbf{P}$ has the form

$$
\{f, g\}=\mathbf{P}^{\mu v} \partial_{\mu} f \partial_{\nu} g .
$$

The bracket (2.5) satisfies the Jacobi identity i.e. $\forall f, g, h \in C^{\infty}(\mathbf{M})$,

$$
\{f,\{g, h\}\}+\{g,\{h, f\}\}+\{h,\{f, g\}\}=[\mathbf{P}, \mathbf{P}]^{\lambda \mu v} \partial_{\lambda} f \partial_{\mu} g \partial_{v} h=0
$$

if $[\mathbf{P}, \mathbf{P}]=0$ and vice versa. A manifold $\mathbf{M}$ equipped with such compatible Poisson structures is called bi-Hamiltonian manifold. If a dynamical system on the manifold $\mathbf{M}$ for which the number of functionally independent integrals of motion $H_{1}, \ldots, H_{n}$ are in bi-involution with respect to this compatible Poisson brackets,

$$
\left\{H_{i}, H_{j}\right\}=\left\{H_{i}, H_{j}\right\}^{\prime}=0
$$

then the system is called bi-Hamiltonian [11]. So to introduce the bi-Hamiltonian structure on the manifold $\mathbf{M}$, we must determine a pair of compatible and independent Poisson bivectors $\mathbf{P}$ and $\mathbf{P}^{\prime}$. 


\subsection{Compatible Poisson structures on Lie groups}

Now we will try to simplify the relations (2.2), (2.3) and (2.4) when $\mathbf{M}$ is a Lie group by using non-coordinate bases.

Definition: [15] In the coordinate basis, $T_{p} \mathbf{M}$ spanned by $\left\{e_{\mu}\right\}=\left\{\partial_{\mu}\right\}$ and $T_{p}^{*} \mathbf{M}$ by $\left\{d x^{\mu}\right\}$, let us consider their linear combinations,

$$
\mathbf{e}_{i}=e_{i}^{\mu} \partial_{\mu}, \quad \theta^{i}=e_{\mu}^{i} d x^{\mu}, \quad\left\{e_{i}^{\mu}\right\} \in G L(m, R)
$$

where det $e_{i}{ }^{\mu}>0$. In other words,$\left\{\mathbf{e}_{i}\right\}$ is the frame of basis vectors which is obtained by a $G L(m, R)$-rotation of the basis $\left\{e_{\mu}\right\}$ and preserving the orientation. In the above $e^{i}{ }_{\mu}$ is inverse of $e_{i}^{\mu}$ and we have

$$
e_{\mu}^{i} e_{i}^{v}=\delta_{\mu}^{v}, e_{\mu}^{i} e_{j}^{\mu}=\delta_{j}^{i}
$$

The bases $\left\{\mathbf{e}_{i}\right\}$ and $\left\{\boldsymbol{\theta}^{i}\right\}$ are called the non-coordinate bases. The coefficients $e_{i}{ }^{\mu}$ are called the vielbeins and we have

$$
\left[\mathbf{e}_{i}, \mathbf{e}_{j}\right]=C_{i j}{ }^{k} \mathbf{e}_{k},
$$

where $C_{i j}{ }^{k}$ is a function of coordinates of the manifold $\mathbf{M}$. When $\mathbf{M}$ is a Lie group $\mathbf{G}$, these coefficients are the structure constants of the Lie algebra $\mathbf{g}$ of the Lie group $\mathbf{G}$ and we have

$$
C_{i j}{ }^{k}=e^{k}{ }_{v}\left(e_{i}^{\mu} \partial_{\mu} e_{j}^{v}-e_{j}^{\mu} \partial_{\mu} e_{i}^{v}\right) \text {. }
$$

Now we write the Poisson structure $\mathbf{P}$ in terms of the non-coordinate basis as ${ }^{\mathrm{a}}$

$$
\mathbf{P}^{\mu v}=e_{i}^{\mu} e_{j}^{v} P^{i j}
$$

Note that in general $P^{i j}$ 's are antisymmetric tensors and functions of the group parameters $x_{i}$ 's. As a first case we consider $P^{i j}$ and $P^{\prime i j}$ as constant antisymmetric matrices

$$
P=\left(\begin{array}{ccccc}
0 & p_{12} & p_{13} & \ldots & p_{1 m} \\
-p_{12} & 0 & p_{23} & \ldots & p_{2 m} \\
-p_{13} & -p_{23} & 0 & \ldots & p_{3 m} \\
\cdot & \cdot & & & \cdot \\
\cdot & \cdot & & & \cdot \\
\cdot & \cdot & & & \cdot \\
-p_{1 m} & -p_{2 m} & -p_{3 m} & \ldots & 0
\end{array}\right), \quad P^{\prime}=\left(\begin{array}{ccccc}
0 & p_{12}^{\prime} & p_{13}^{\prime} & \ldots & p_{1 m}^{\prime} \\
-p_{12}^{\prime} & 0 & p_{23}^{\prime} & \ldots & p_{2 m}^{\prime} \\
-p_{13}^{\prime} & -p_{23}^{\prime} & 0 & \ldots & p_{3 m}^{\prime} \\
\cdot & \cdot & & \\
\cdot & \cdot & & & \cdot \\
\cdot & \cdot & & & \cdot \\
-p_{1 m}^{\prime} & -p_{2 m}^{\prime} & -p_{3 m}^{\prime} & \ldots & 0
\end{array}\right)
$$

where $p_{i j}$ and $p_{i j}^{\prime}$ are real constants. Now using (2.1), (2.11) and (2.12) one can rewrite the relations (2.1) with (2.2), (2.3) and (2.4) as follows:

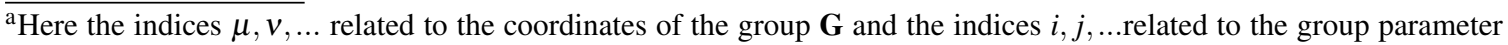
$\left\{x_{i}\right\}$. Furthermore, in the following we will consider $\mathbf{G}$ as a phase space of the dynamical system, hence $m=2 n$ must be even.
} 


$$
\begin{gathered}
C_{k i}{ }^{s} P^{k z} P^{i \gamma}+C_{k j}{ }^{\gamma} P^{k z} P^{s j}+C_{k i}{ }^{z} P^{k \gamma} P^{i s}=0, \\
C_{k i}{ }^{s} P^{\prime k z} P^{\prime i \gamma}+C_{k j}{ }^{\gamma} P^{\prime k z} P^{\prime s j}+C_{k i}{ }^{z} P^{\prime k \gamma} P^{\prime i s}=0, \\
C_{k i}{ }^{s}\left(P^{k z} P^{\prime i \gamma}+P^{\prime k z} P^{i \gamma}\right)+C_{k j}^{\gamma}\left(P^{k z} P^{\prime s j}+P^{\prime k z} P^{s j}\right)+C_{k i}{ }^{z}\left(P^{k \gamma} P^{\prime i s}+P^{\prime k \gamma} P^{i s}\right)=0,
\end{gathered}
$$

then using the adjoint representation of the Lie algebra g, i.e.: $\left(\mathscr{X}_{i}\right)_{j}{ }_{j}=-C_{i j}{ }^{k}$ and $\left(\mathscr{Y}^{k}\right)_{i j}=-C_{i j}{ }^{k}$, one can rewrite the above relations in the following matrix forms: ${ }^{b}$

$$
\begin{gathered}
P \mathscr{X}_{i} P^{i \gamma}+P \mathscr{Y}^{\gamma} P+P^{i \gamma} \mathscr{X}_{i}^{t} P=0, \\
P^{\prime} \mathscr{X}_{i} P^{i \gamma}+P^{\prime} \mathscr{Y}^{\gamma} P^{\prime}+P^{i \gamma} \mathscr{X}_{i}^{t} P^{\prime}=0, \\
P \mathscr{X}_{i} P^{\prime i \gamma}+P^{\prime} \mathscr{X}_{i} P^{i \gamma}+P \mathscr{Y}^{\gamma} P^{\prime}+P^{\prime} \mathscr{Y}^{\gamma} P+P^{i \gamma} \mathscr{X}_{i}^{t} P^{\prime}+P^{\prime i \gamma} \mathscr{X}_{i}^{t} P=0 .
\end{gathered}
$$

In this way having the structure constants of the Lie algebra $\mathbf{g}$, one can solve the matrix equations (2.17) - (2.19) in order to obtain $P$ and $P^{\prime}$. Here we will consider four dimensional real Lie algebras [17]. For all of the symplectic ${ }^{c}$ four dimensional real Lie algebras [16], one can see that all the solutions of (2.17) - (2.19) are equivalent.

For this reason as a second case we consider $P^{i j}$ as (2.13) but $P^{\prime i j}$ as a linear functions of group parameters $x_{i}$ of the Lie group $\mathbf{G}$ as follows:

$$
P^{\prime}=\left(\begin{array}{cccc}
0 & p_{12}^{\prime}+\sum_{i=1}^{m} a_{2 i} x_{i} & p_{13}^{\prime}+\sum_{i=1}^{m} a_{3 i} x_{i} & \ldots p_{1 m}^{\prime}+\sum_{i=1}^{m} a_{m i} x_{i} \\
-p_{12}^{\prime}-\sum_{i=1}^{m} a_{2 i} x_{i} & 0 & p_{23}^{\prime}+\sum_{i=1}^{m} b_{3 i} x_{i} & \ldots p_{2 m}^{\prime}+\sum_{i=1}^{m} b_{m i} x_{i} \\
-p_{13}^{\prime}-\sum_{i=1}^{m} a_{3 i} x_{i}-p_{23}^{\prime}-\sum_{i=1}^{m} b_{3 i} x_{i} & 0 & \ldots p_{3 m}^{\prime}+\sum_{i=1}^{4} c_{m i} x_{i} \\
\cdot & \cdot & \cdot & \cdot \\
\cdot & \cdot & \cdot & \cdot \\
\cdot & \cdot & \cdot & \\
-p_{1 m}^{\prime}-\sum_{i=1}^{m} a_{m i} x_{i}-p_{2 m}^{\prime}-\sum_{i=1}^{m} b_{m i} x_{i}-p_{3 m}^{\prime}-\sum_{i=1}^{4} c_{m i} x_{i} \ldots & 0
\end{array}\right)
$$

where $p_{i j}^{\prime}$ and $a_{i j}$ 's are real constants; then relations (2.1) with (2.3) and (2.4) have the following matrix forms:

\footnotetext{
${ }^{\mathrm{b}}$ Here the upper index $t$ represents the transpose of a matrix.

${ }^{c}$ Note that here we will consider symplectic four dimensional real Lie algebras [1] and not all of them [17], because we will construct integrable systems over these related Lie groups.
} 


$$
P^{\prime} \mathscr{X}_{i} P^{\prime i \gamma}+P^{\prime} \mathscr{Y}^{\gamma} P^{\prime}+P^{\prime i \gamma} \mathscr{X}_{i}^{t} P^{\prime}+\left(e^{t} P^{\prime}\right)^{k \gamma} \partial_{k} P^{\prime}+A+B=0,
$$

$$
P \mathscr{X}_{i} P^{\prime i \gamma}+P^{\prime} \mathscr{X}_{i} P^{i \gamma}+P \mathscr{Y}^{\gamma} P^{\prime}+P^{\prime} \mathscr{Y}^{\gamma} P+P^{i \gamma} \mathscr{X}_{i}^{t} P^{\prime}+P^{\prime i \gamma} \mathscr{X}_{i}^{t} P+\left(e^{t} P\right)^{k \gamma} \partial_{k} P^{\prime}+A^{\prime}+B^{\prime}=0,
$$

where $e^{t}$ is a transpose of the vielbein $e_{\alpha}^{\mu}$ and $A, B, A^{\prime}$ and $B^{\prime}$ have the following forms:

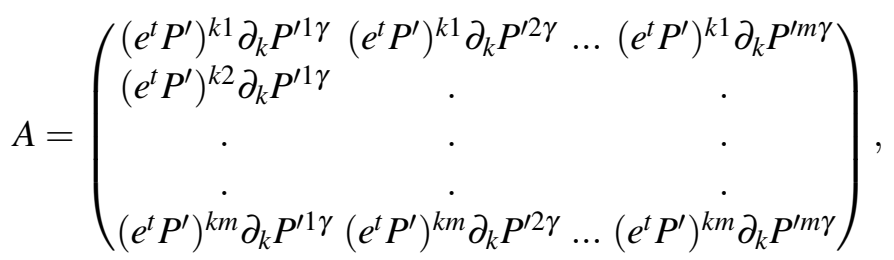

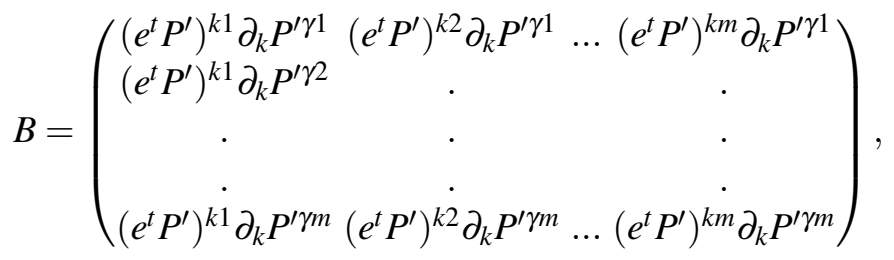

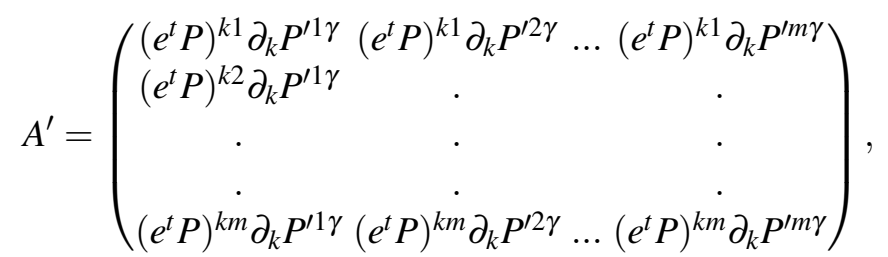

$$
\begin{aligned}
& B^{\prime}=\left(\begin{array}{cccc}
\left(e^{t} P\right)^{k 1} \partial_{k} P^{\prime \gamma 1} & \left(e^{t} P\right)^{k 2} \partial_{k} P^{\prime \gamma 1} & \ldots & \left(e^{t} P\right)^{k m} \partial_{k} P^{\prime \gamma 1} \\
\left(e^{t} P\right)^{k 1} \partial_{k} P^{\prime \gamma 2} & \cdot & \cdot \\
\cdot & \cdot & \cdot \\
\cdot & \cdot & \cdot \\
\left(e^{t} P\right)^{k 1} \partial_{k} P^{\prime \gamma m} & \left(e^{t} P\right)^{k 2} \partial_{k} P^{\prime \gamma m} & \ldots & \left(e^{t} P\right)^{k m} \partial_{k} P^{\prime \gamma m}
\end{array}\right) .
\end{aligned}
$$

Now we will try to solve (2.17), (2.21) and (2.22) for four dimensional real Lie groups.

\section{Some compatible Poisson structures on symplectic four dimensional real Lie groups}

Having the structure constants of the Lie algebra g, we will solve matrix equations (2.17), (2.21) and (2.22) in order to obtain $P(2.13)$ and $P^{\prime}(2.20)$. For completeness the list of symplectic four dimensional real Lie algebras [1] are brought in appendix A.

Let us consider an example; for Lie algebra $A_{4,1}$ we have the following non zero commutators and the matrices $\mathscr{X}_{i}$ and $\mathscr{Y}^{i}$ :

$$
\begin{gathered}
{\left[\mathbf{e}_{2}, \mathbf{e}_{4}\right]=\mathbf{e}_{1},\left[\mathbf{e}_{3}, \mathbf{e}_{4}\right]=\mathbf{e}_{2},} \\
\mathscr{X}_{1}=\left(\begin{array}{llll}
0 & 0 & 0 & 0 \\
0 & 0 & 0 & 0 \\
0 & 0 & 0 & 0 \\
0 & 0 & 0 & 0
\end{array}\right), \mathscr{X}_{2}=\left(\begin{array}{cccc}
0 & 0 & 0 & 0 \\
0 & 0 & 0 & 0 \\
0 & 0 & 0 & 0 \\
-1 & 0 & 0 & 0
\end{array}\right), \mathscr{X}_{3}=\left(\begin{array}{cccc}
0 & 0 & 0 & 0 \\
0 & 0 & 0 & 0 \\
0 & 0 & 0 & 0 \\
0 & -1 & 0 & 0
\end{array}\right), \mathscr{X}_{4}=\left(\begin{array}{llll}
0 & 0 & 0 & 0 \\
1 & 0 & 0 & 0 \\
0 & 1 & 0 & 0 \\
0 & 0 & 0 & 0
\end{array}\right),
\end{gathered}
$$




$$
\mathscr{Y}^{1}=\left(\begin{array}{cccc}
0 & 0 & 0 & 0 \\
0 & 0 & 0 & -1 \\
0 & 0 & 0 & 0 \\
0 & 1 & 0 & 0
\end{array}\right), \mathscr{Y}^{2}=\left(\begin{array}{cccc}
0 & 0 & 0 & 0 \\
0 & 0 & 0 & 0 \\
0 & 0 & 0 & -1 \\
0 & 0 & 1 & 0
\end{array}\right), \mathscr{Y}^{3}=\left(\begin{array}{llll}
0 & 0 & 0 & 0 \\
0 & 0 & 0 & 0 \\
0 & 0 & 0 & 0 \\
0 & 0 & 0 & 0
\end{array}\right), \mathscr{Y}^{4}=\left(\begin{array}{llll}
0 & 0 & 0 & 0 \\
0 & 0 & 0 & 0 \\
0 & 0 & 0 & 0 \\
0 & 0 & 0 & 0
\end{array}\right)
$$

also according to [13] for Lie algebra $A_{4,1}$ the matrix $e_{\alpha}^{\mu}$ has the following form:

$$
\left(e_{\alpha}^{\mu}\right)=\left(\begin{array}{cccc}
1 & 0 & 0 & 0 \\
x_{4} & 1 & 0 & 0 \\
x_{4}^{2} / 2 & x_{4} & 1 & 0 \\
0 & 0 & 0 & 1
\end{array}\right)
$$

inserting $\mathscr{X}_{i}, \mathscr{Y}^{i}$ and $e_{\alpha}^{\mu}$ in (2.17), (2.21) and (2.22) one can obtain the compatible Poisson structure $P$ and $P^{\prime}$ for the Lie algebra $A_{4,1}$. One of the solutions has the following forms:

$$
P=\left(\begin{array}{cccc}
0 & p_{12} & 0 & p_{14} \\
* & 0 & p_{23} & 0 \\
* & * & 0 & 0 \\
* & * & * & 0
\end{array}\right), P^{\prime}=\left(\begin{array}{cccc}
0 & p_{12}^{\prime}-\left(a_{44}+p_{24}^{\prime}\right) x_{2}+a_{23} x_{3}+a_{24} x_{4} & p_{13}^{\prime}+a_{44} x_{3} & a_{44} x_{4} \\
* & 0 & 0 & p_{24}^{\prime} \\
* & * & 0 & 0 \\
* & * & * & 0
\end{array}\right)
$$

Here $p_{12}, p_{14}, p_{23}, p_{12}^{\prime}, p_{13}^{\prime}, p_{24}^{\prime}, a_{23}, a_{44}$ and $a_{24}$ arbitrary real constants. In this way we have obtained some compatible Poisson structure ( $P$ is constant and $P^{\prime}$ is linear function of Lie group coordinates) on symplectic four dimensional real Lie algebras, the results being summarized in Table 1 . Note that all parameters $a_{i j}, p_{i j}$ and $p_{i j}^{\prime}$ are arbitrary real constants.

\section{Integrable bi-Hamiltonian systems on symplectic four dimensional real Lie groups}

In this section, we will construct the integrable bi-Hamiltonian systems with four dimensional real Lie groups as phase space. For this purpose, in the previous section we consider four dimensional real Lie groups such that they have symplectic structures [16], [1]. Here, we will construct the models on these Lie groups separately as follows.

An important class of bi-Hamiltonian manifold occurs when one of the compatible Poisson structures is invertible i.e., the Poisson bracket $\{.,$.$\} associated with \mathbf{P}$ is invertible. Then one can define a linear map $\mathbf{N}: T M \longrightarrow T M$ acting on the tangent bundle by [11]

$$
\mathbf{N}=\mathbf{P}^{\prime} \mathbf{P}^{-1}
$$

Theorem (Magri-Morosi): [12], [8] A remarkable consequence of the compatibility of $\mathbf{P}$ and $\mathbf{P}^{\prime}$ is that the torsion of Nijenhuis tensor $\mathbf{N}$

$$
T_{\mathbf{N}}(X, Y)=[\mathbf{N} X, \mathbf{N} Y]-\mathbf{N}[\mathbf{N} X, Y]-\mathbf{N}[X, \mathbf{N} Y]+\mathbf{N}^{2}[X, Y]
$$

identically vanishes; where $X$ and $Y$ are arbitrary vector fields and the bracket $[X, Y]$ denotes the Lie bracket (commutator). One of the main properties of $\mathbf{N}$ is that the normalized traces of the 
J. Abedi-Fardad et al. / Compatible Poisson structures and integrable bi-Hamiltonian systems

Table 1: Compatible Poisson structure on symplectic four dimensional real Lie algebras.

\begin{tabular}{|c|c|c|c|}
\hline $\mathbf{g}$ & $P$ & $P^{\prime}$ & Comments \\
\hline$A_{4,1}$ & $\left(\begin{array}{cccc}0 & p_{12} & 0 & p_{14} \\
* & 0 & p_{23} & 0 \\
* & * & 0 & 0 \\
* & * & * & 0\end{array}\right)$ & $\left(\begin{array}{cccc}0 & p_{12}^{\prime}-\left(a_{44}+p_{24}^{\prime}\right) x_{2}+a_{23} x_{3}+a_{24} x_{4} & p_{13}^{\prime}+a_{44} x_{3} & a_{44} x_{4} \\
* & 0 & 0 & p_{24}^{\prime} \\
* & * & 0 & 0 \\
* & * & * & 0\end{array}\right)$ & $p_{14} p_{23} \neq 0$ \\
\hline$A_{4,2}^{-1}$ & $\left(\begin{array}{cccc}0 & p_{12} & p_{13} & 0 \\
* & 0 & p_{23} & p_{24} \\
* & * & 0 & 0 \\
* & * & * & 0\end{array}\right)$ & $\left(\begin{array}{cccc}0 & p_{12}^{\prime}+a_{23} x_{3}+a_{24} x_{4} & \frac{a_{53} p_{13}}{p_{23}} x_{3} & 0 \\
* & 0 & a_{53} x_{3} & p_{24}^{\prime}+a_{64} x_{4} \\
* & * & 0 & 0 \\
* & * & * & 0\end{array}\right)$ & $p_{13} p_{24} \neq 0$ \\
\hline$A_{4,3}$ & $\left(\begin{array}{cccc}0 & p_{12} & 0 & p_{14} \\
* & 0 & p_{23} & 0 \\
* & * & 0 & 0 \\
* & * & * & 0\end{array}\right)$ & $\left(\begin{array}{cccc}0 p_{12}^{\prime}+a_{23} x_{3}+a_{24} x_{4} & a_{33} x_{3} & a_{33} x_{4} \\
* & 0 & a_{53} x_{3}+\frac{a_{33} p_{23}}{p_{14}} x_{4} & 0 \\
* & * & 0 & 0 \\
* & * & * & 0\end{array}\right)$ & $p_{14} p_{23} \neq 0$ \\
\hline$A_{4,5}^{a,-a}$ & $\left(\begin{array}{cccc}0 & 0 & p_{13} & p_{14} \\
* & 0 & p_{23} & 0 \\
* & * & 0 & 0 \\
* & * & * & 0\end{array}\right)$ & $\left(\begin{array}{cccc}0 & 0 & p_{13}^{\prime}+a_{32} x_{2}+a_{34} x_{4} & a_{44} x_{4} \\
* 0 & p_{23}^{\prime}+a_{52} x_{2} & 0 \\
* * & 0 & 0 \\
* * & * & 0\end{array}\right)$ & $p_{14} p_{23} \neq 0$ \\
\hline$A_{4,5}^{-1,-1}$ & $\left(\begin{array}{cccc}0 & p_{12} & p_{13} & 0 \\
* & 0 & p_{23} & p_{24} \\
* & * & 0 & 0 \\
* & * & * & 0\end{array}\right)$ & $\left(\begin{array}{cccc}0 & p_{12}^{\prime}+a_{23} x_{3}+a_{24} x_{4} & \frac{a_{53} p_{13} x_{3}}{p_{23}} & 0 \\
* & 0 & a_{53} x_{3} & a_{64} x_{4} \\
* & * & 0 & 0 \\
* & * & * & 0\end{array}\right)$ & $p_{13} p_{24} \neq 0$ \\
\hline$A_{4,6}^{a, 0}$ & $\left(\begin{array}{cccc}0 & 0 & 0 & p_{14} \\
* & 0 & p_{23} & 0 \\
* & * & 0 & 0 \\
* & * & * & 0\end{array}\right)$ & 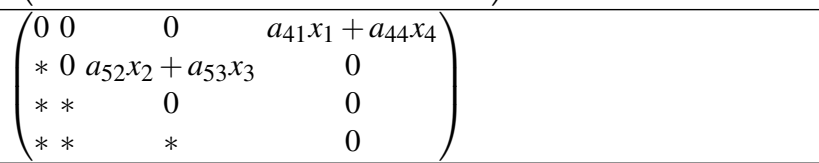 & $p_{14} p_{23} \neq 0$ \\
\hline$A_{4,9}^{0}$ & $\left(\begin{array}{cccc}0 & 0 & p_{13} & p_{23} \\
* & 0 & p_{23} & 0 \\
* & * & 0 & 0 \\
* & * & * & 0\end{array}\right)$ & 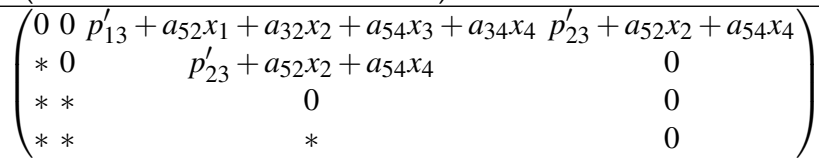 & $p_{23} \neq 0$ \\
\hline$A_{4,12}$ & $\left(\begin{array}{cccc}0 & 0 & 0 & p_{14} \\
* & 0 & -p_{14} & 0 \\
* & * & 0 & 0 \\
* & * & * & 0\end{array}\right)$ & 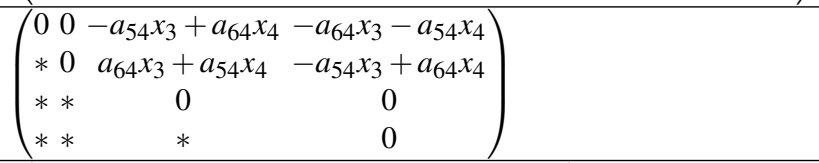 & $p_{14} \neq 0$ \\
\hline$I I \oplus R$ & $\left(\begin{array}{cccc}0 & p_{12} & p_{13} & 0 \\
* & 0 & 0 & 0 \\
* & * & 0 & p_{34} \\
* & * & * & 0\end{array}\right)$ & $\left(\begin{array}{cccc}0 & a_{44} x_{2} & p_{13}^{\prime}+a_{32} x_{2}-\left(a_{44}+p_{23}^{\prime}\right) x_{3} & a_{44} x_{4} \\
* & 0 & p_{23}^{\prime} & 0 \\
* & * & 0 & 0 \\
* & * & * & 0\end{array}\right)$ & $p_{12} p_{34} \neq 0$ \\
\hline$I I I \oplus R$ & $\left(\begin{array}{cccc}0 & p_{13} & p_{13} & 0 \\
* & 0 & 0 & p_{24} \\
* & * & 0 & 0 \\
* & * & * & 0\end{array}\right)$ & $\left(\begin{array}{cccc}0 & a_{33} x_{3} & a_{33} x_{3} & 0 \\
* & 0 & 0 & a_{64} x_{4} \\
* & * & 0 & 0 \\
* & * & * & 0\end{array}\right)$ & $p_{13} p_{24} \neq 0$ \\
\hline$V I_{0} \oplus R$ & $\left(\begin{array}{cccc}0 & p_{12} & 0 & 0 \\
* & 0 & 0 & 0 \\
* & * & 0 & p_{34} \\
* & * & * & 0\end{array}\right)$ & $\left(\begin{array}{cccc}0 & p_{12}^{\prime}+a_{22} x_{1}+a_{22} x_{2} & 0 & 0 \\
* & 0 & 0 & 0 \\
* & * & 0 & p_{34}^{\prime}+a_{73} x_{3}+a_{74} x_{4} \\
* & * & * & 0\end{array}\right)$ & $p_{12} p_{34} \neq 0$ \\
\hline$V I I_{0} \oplus R$ & $\left(\begin{array}{cccc}0 & p_{12} & 0 & 0 \\
* & 0 & 0 & 0 \\
* & * & 0 & p_{34} \\
* & * & * & 0\end{array}\right)$ & $\left(\begin{array}{cccc}0 & a_{21} x_{1}+a_{22} x_{2} & 0 & 0 \\
* & 0 & 0 & 0 \\
* & * & 0 & a_{73} x_{3}+a_{74} x_{4} \\
* & * & * & 0\end{array}\right)$ & $p_{12} p_{34} \neq 0$ \\
\hline$A_{2} \oplus A_{2}$ & $\left(\begin{array}{cccc}0 & p_{12} & 0 & 0 \\
* & 0 & 0 & p_{24} \\
* & * & 0 & p_{34} \\
* & * & * & 0\end{array}\right)$ & $\left(\begin{array}{cclc}0 & a_{21} x_{1} & 0 & 0 \\
* & 0 & 0 & p_{24}^{\prime}+a_{61} x_{1}+a_{63} x_{3} \\
* & * & 0 & a_{73} x_{3} \\
* & * & * & 0\end{array}\right)$ & $p_{12} p_{34} \neq 0$ \\
\hline
\end{tabular}


powers of $\mathbf{N}$ are integrals of motion

$$
H_{k}=\frac{1}{2 k} \operatorname{Tr} \mathbf{N}^{k}
$$

and satisfying Lenard-Magri recurrent relations [11]

$$
\mathbf{P}^{\prime} d H_{i}=\mathbf{P} d H_{i+1} .
$$

In Table 1 , the matrices $P$ and $P^{\prime}$ have been given for the various symplectic Lie algebras. Now inserting $P$ and $P^{\prime}$ in (2.12) and using the related vielbeins [13] the compatible Poisson structures $\mathbf{P}$ and $\mathbf{P}^{\prime}$ on Lie groups are obtained, then using (4.1) and (4.3) one can find the Hamiltonian and integrals of motions of bi-Hamiltonian systems. In the following we will perform this work separately for symplectic four dimensional real Lie groups. In this way we obtain new bi-Hamiltonian systems over four dimensional real Lie groups as phase spaces.

\section{Lie group $\mathbf{A}_{4,1}$ :}

Inserting $P$ and $P^{\prime}$ in (2.12) one can obtain the compatible Poisson structures $\mathbf{P}$ and $\mathbf{P}^{\prime}$ on the Lie group $\mathbf{A}_{4,1}$ as follows:

$$
\begin{gathered}
\mathbf{P}=\left(\begin{array}{cccc}
0 & p_{12}+\frac{p_{23} x_{4}^{2}}{2} & p_{23} x_{4} & p_{14} \\
* & 0 & p_{23} & 0 \\
* & * & 0 & 0 \\
* & * & * & 0
\end{array}\right), \\
\mathbf{P}^{\prime}=\left(\begin{array}{llll}
0 & a_{23} x_{3}-\left(a_{44}+p_{24}^{\prime}\right) x_{2}+a_{24} x_{4}+p_{13}^{\prime} x_{4}+a_{44} x_{3} x_{4} & p_{13}^{\prime}+a_{44} x_{3} & \left(p_{24}^{\prime}+a_{44}\right) x_{4} \\
* & 0 & 0 & p_{24}^{\prime} \\
* & * & 0 & 0 \\
* & * & * & 0
\end{array}\right) .
\end{gathered}
$$

Now by means of (4.1) and (4.3), the integrals of motion can be found for this Lie group as follows:

$$
H_{1}=\frac{a_{44} x_{4}}{p_{14}}, H_{2}=\frac{2 p_{14} p_{24}^{\prime}\left(p_{13}^{\prime}+a_{44} x_{3}\right)+a_{44}^{2} p_{23} x_{4}^{2}}{2 p_{14}^{2} p_{23}} .
$$

Lie group $\mathbf{A}_{4,2}^{-1}$ :

Inserting $P$ and $P^{\prime}$ in (2.12) one can obtain the compatible Poisson structures $\mathbf{P}$ and $\mathbf{P}^{\prime}$ on the Lie group $\mathbf{A}_{4,2}^{-1}$ as follows:

$$
\begin{gathered}
\mathbf{P}=\left(\begin{array}{cccc}
0 & p_{12}+p_{13} x_{4} & p_{13} & 0 \\
* & 0 & e^{2 x_{4}} p_{23} & e^{x_{4}} p_{24} \\
* & * & 0 & 0 \\
* & * & * & 0
\end{array}\right), \\
\mathbf{P}^{\prime}=\left(\begin{array}{ccccc}
0 & p_{12}^{\prime}+a_{23} x_{3}+a_{24} x_{4}+\frac{a_{53} p_{13} x_{3} x_{4}}{p_{23}} & \frac{a_{53} p_{13} x_{3}}{p_{23}} & 0 \\
* & 0 & & a_{53} e^{2 x_{4}} x_{3} & e^{x_{4}} \\
* & * & 0 & \left.p_{24}^{\prime}+a_{64} x_{4}\right) \\
* & * & & 0 & 0
\end{array}\right) .
\end{gathered}
$$


By means of (4.1) and (4.3), the integrals of motion can be found for this Lie group as follows:

$$
H_{1}=\frac{p_{23} p_{24}^{\prime}+a_{53} p_{24} x_{3}+a_{64} p_{23} x_{4}}{p_{23} p_{24}}, H_{2}=\frac{1}{2}\left(\frac{a_{53}^{2} x_{3}^{2}}{p_{23}^{2}}+\frac{\left(p_{24}^{\prime}+a_{64} x_{4}\right)^{2}}{p_{24}^{2}}\right) .
$$

\section{Lie group $\mathbf{A}_{4,3}$ :}

Inserting $P$ and $P^{\prime}$ in (2.12) one can obtain the compatible Poisson structures $\mathbf{P}$ and $\mathbf{P}^{\prime}$ on the Lie group $\mathbf{A}_{4,3}$ as follows:

$$
\mathbf{P}=\left(\begin{array}{cccc}
0 & p_{12} e^{x_{4}} & 0 & p_{14} e^{x_{4}} \\
* & 0 & p_{23} & 0 \\
* & * & 0 & 0 \\
* & * & * & 0
\end{array}\right), \mathbf{P}^{\prime}=\left(\begin{array}{cccc}
0 e^{x_{4}}\left(p_{12}^{\prime}+a_{23} x_{3}+a_{24} x_{4}+a_{33} x_{3} x_{4}\right) & a_{33} e^{x_{4}} x_{3} & a_{33} e^{x_{4}} x_{4} \\
* & 0 & a_{53} x_{3}+\frac{a_{33} p_{23} x_{4}}{p_{14}} & 0 \\
* & * & 0 & 0 \\
* & * & * & 0
\end{array}\right)
$$

Now by means of (4.1) and (4.3), the integrals of motion can be found for this Lie group as follows:

$$
H_{1}=\frac{a_{53} x_{3}}{p_{23}}+\frac{2 a_{33} x_{4}}{p_{14}}, H_{2}=\frac{1}{2}\left(\frac{a_{33}^{2} x_{4}^{2}}{p_{14}^{2}}+\left(\frac{a_{53} x_{3}}{p_{23}}+\frac{a_{33} x_{4}}{p_{14}}\right)^{2}\right) .
$$

\section{Lie group $\mathbf{A}_{4,5}^{a,-a}$ :}

Inserting $P$ and $P^{\prime}$ in (2.12) one can obtain the compatible Poisson structures $\mathbf{P}$ and $\mathbf{P}^{\prime}$ on the Lie group $\mathbf{A}_{4,5}^{a,-a}$ as follows:

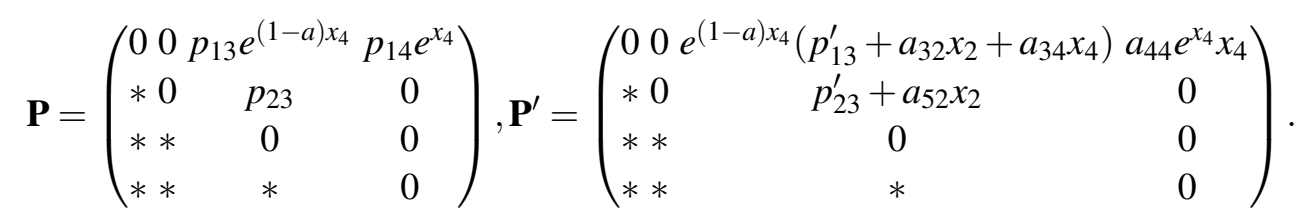

Now by means of (4.1) and (4.3), the integrals of motion can be found for this Lie group as follows:

$$
H_{1}=\frac{p_{23}^{\prime}+a_{52} x_{2}}{p_{23}}+\frac{a_{44} x_{4}}{p_{14}}, H_{2}=\frac{1}{2}\left(\frac{\left(p_{23}^{\prime}+a_{52} x_{2}\right)^{2}}{p_{23}^{2}}+\frac{a_{44}^{2} x_{2}^{2}}{p_{14}^{2}}\right) .
$$

Lie group $\mathbf{A}_{4,5}^{-1,-1}$ :

Inserting $P$ and $P^{\prime}$ in (2.12) one can obtain the compatible Poisson structures $\mathbf{P}$ and $\mathbf{P}^{\prime}$ on the Lie group $\mathbf{A}_{4,5}^{-1,-1}$ as follows:

$$
\mathbf{P}=\left(\begin{array}{cccc}
0 & p_{12} & p_{13} & 0 \\
* & 0 & p_{23} e^{-2 x_{4}} & p_{24} e^{-x_{4}} \\
* & * & 0 & 0 \\
* & * & * & 0
\end{array}\right), \mathbf{P}^{\prime}=\left(\begin{array}{cccc}
0 & p_{12}^{\prime}+a_{23} x_{3}+a_{24} x_{4} & \frac{a_{53} p_{13} x_{3}}{p_{23}} & 0 \\
* & 0 & a_{53} e^{-2 x_{4}} x_{3} & a_{64} e^{-x_{4}} x_{4} \\
* & * & 0 & 0 \\
* & * & * & 0
\end{array}\right)
$$

Now by means of (4.1) and (4.3), the integrals of motion can be found for this Lie group as follows:

$$
H_{1}=\frac{a_{53} x_{3}}{p_{23}}+\frac{a_{64} x_{4}}{p_{24}}, H_{2}=\frac{1}{2}\left(\left(\frac{a_{53} x_{3}}{p_{23}}\right)^{2}+\left(\frac{a_{64} x_{4}}{p_{24}}\right)^{2}\right) .
$$

Lie group $\mathbf{A}_{4,6}^{a, 0}$ :

Inserting $P$ and $P^{\prime}$ in (2.12) one can obtain the compatible Poisson structures $\mathbf{P}$ and $\mathbf{P}^{\prime}$ on the Lie 
group $\mathbf{A}_{4,6}^{a, 0}$ as follows:

$$
\mathbf{P}=\left(\begin{array}{cccc}
0 & 0 & 0 & p_{14} e^{a x_{4}} \\
* & 0 & p_{23} & 0 \\
* * & 0 & 0 \\
* * & * & 0
\end{array}\right), \mathbf{P}^{\prime}=\left(\begin{array}{cccc}
0 & 0 & 0 & e^{a x_{4}}\left(a_{41} x_{1}+a_{44} x_{4}\right) \\
* 0 & a_{52} x_{2}+a_{53} x_{3} & 0 \\
* * & 0 & 0 \\
* * & * & 0
\end{array}\right)
$$

Now by means of (4.1) and (4.3), the integrals of motion can be found for this Lie group as follows:

$$
H_{1}=\frac{a_{41} x_{1}+a_{44} x_{4}}{p_{14}}+\frac{a_{52} x_{2}+a_{53} x_{3}}{p_{23}}, H_{2}=\frac{1}{2}\left(\left(\frac{a_{41} x_{1}+a_{44} x_{4}}{p_{14}}\right)^{2}+\left(\frac{a_{52} x_{2}+a_{53} x_{3}}{p_{23}}\right)^{2}\right) .
$$

Lie group $\mathbf{A}_{4,12}$ :

Inserting $P$ and $P^{\prime}$ in (2.12) one can obtain the compatible Poisson structures $\mathbf{P}$ and $\mathbf{P}^{\prime}$ on the Lie group $\mathbf{A}_{4,12}$ as follows:

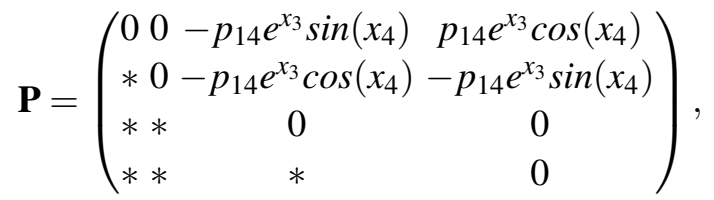

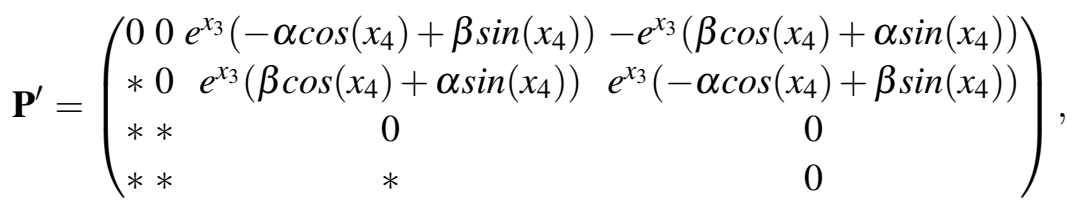

where $\alpha=a_{54} x_{3}-a_{64} x_{4}$ and $\beta=a_{64} x_{3}+a_{54} x_{4}$. Now by means of (4.1) and (4.3), the integrals of motion can be found for this Lie group as follows:

$$
H_{1}=\frac{-2 a_{64} x_{3}-2 a_{54} x_{4}}{p_{14}}, H_{2}=\frac{\left(a_{64}^{2}-a_{54}^{2}\right) x_{3}^{2}+4 a_{54} a_{64} x_{3} x_{4}+\left(a_{54}^{2}-a_{64}^{2}\right) x_{4}^{2}}{p_{14}^{2}} .
$$

Lie group $\mathbf{A}_{\mathbf{2}} \oplus \mathbf{A}_{\mathbf{2}}$ :

Inserting $P$ and $P^{\prime}$ in (2.12) one can obtain the compatible Poisson structures $\mathbf{P}$ and $\mathbf{P}^{\prime}$ on the Lie group $\mathbf{A}_{2} \oplus A_{2}$ as follows:

$$
\mathbf{P}=\left(\begin{array}{cccc}
0 & p_{12} & 0 & 0 \\
* & 0 & 0 & p_{24} \\
* & * & 0 & p_{34} \\
* & * & * & 0
\end{array}\right), \mathbf{P}^{\prime}=\left(\begin{array}{ccccc}
0 & a_{21} x_{1} & 0 & 0 \\
* & 0 & 0 & p_{24}^{\prime}+a_{61} x_{1}+a_{63} x_{3} \\
* & * & 0 & a_{73} x_{3} \\
* & * & * & 0
\end{array}\right)
$$

Now by means of (4.1) and (4.3), the integrals of motion can be found for this Lie group as follows:

$$
H_{1}=\frac{a_{21} x_{1}}{p_{12}}+\frac{a_{73} x_{3}}{p_{34}}, H_{2}=\frac{a_{21}^{2} x_{1}^{2}}{2 p_{12}^{2}}+\frac{a_{73}^{2} x_{3}^{2}}{2 p_{34}^{2}} .
$$

\section{Lie group $\mathrm{VII}_{0} \oplus \mathbf{R}$ :}

Inserting $P$ and $P^{\prime}$ in (2.12) one can obtain the compatible Poisson structures $\mathbf{P}$ and $\mathbf{P}^{\prime}$ on the Lie 
group $\mathbf{V I I}_{\mathbf{0}} \oplus \mathbf{R}$ as follows:

$$
\mathbf{P}=\left(\begin{array}{cccc}
0 & p_{12} & 0 & 0 \\
* & 0 & 0 & 0 \\
* & * & 0 & p_{34} \\
* & * & * & 0
\end{array}\right), \mathbf{P}^{\prime}=\left(\begin{array}{cccc}
0 & a_{21} x_{1}+a_{22} x_{2} & 0 & 0 \\
* & 0 & 0 & 0 \\
* & * & 0 & a_{73} x_{3}+a_{74} x_{4} \\
* & * & * & 0
\end{array}\right)
$$

Now by means of (4.1) and (4.3), the integrals of motion can be found for this Lie group as follows:

$$
H_{1}=\frac{a_{21} x_{1}+a_{22} x_{2}}{p_{12}}+\frac{a_{73} x_{3}+a_{74} x_{4}}{p_{34}}, H_{2}=\frac{1}{2}\left(\left(\frac{a_{21} x_{1}+a_{22} x_{2}}{p_{12}}\right)^{2}+\left(\frac{a_{73} x_{3}+a_{74} x_{4}}{p_{34}}\right)^{2}\right) .
$$

\section{Lie group $\mathbf{V I}_{\mathbf{0}} \oplus \mathbf{R}$ :}

Inserting $P$ and $P^{\prime}$ in (2.12) one can obtain the compatible Poisson structures $\mathbf{P}$ and $\mathbf{P}^{\prime}$ on the Lie group $\mathbf{V I}_{\mathbf{0}} \oplus \mathbf{R}$ as follows:

$$
\mathbf{P}=\left(\begin{array}{cccc}
0 & p_{12} & 0 & 0 \\
* & 0 & 0 & 0 \\
* & * & 0 & p_{34} \\
* & * & * & 0
\end{array}\right), \mathbf{P}^{\prime}=\left(\begin{array}{cclc}
0 & p_{12}^{\prime}+a_{22}\left(x_{1}+x_{2}\right) & 0 & 0 \\
* & 0 & 0 & 0 \\
* & * & 0 & \left.p_{34}^{\prime}+a_{73} x_{3}+a_{74} x_{4}\right) \\
* & * & * & 0
\end{array}\right)
$$

Now by means of (4.1) and (4.3), the integrals of motion can be found for this Lie group as follows:

$$
\begin{array}{r}
H_{1}=\frac{p_{12}^{\prime}+a_{22} x_{1}+a_{22} x_{2}}{p_{12}}+\frac{p_{34}^{\prime}+a_{73} x_{3}+a_{74} x_{4}}{p_{34}}, \\
H_{2}=\frac{1}{2}\left(\left(\frac{p_{12}^{\prime}+a_{22} x_{1}+a_{22} x_{2}}{p_{12}}\right)^{2}+\left(\frac{p_{34}^{\prime}+a_{73} x_{3}+a_{74} x_{4}}{p_{34}}\right)^{2}\right) .
\end{array}
$$

\section{Lie group III $\oplus \mathbf{R}$ :}

Inserting $P$ and $P^{\prime}$ in (2.12) one can obtain the compatible Poisson structures $\mathbf{P}$ and $\mathbf{P}^{\prime}$ on the Lie group III $\oplus \mathbf{R}$ as follows:

$$
\mathbf{P}=\left(\begin{array}{cccc}
0 & p_{13} & p_{13} & 0 \\
* & 0 & 0 & p_{24} \\
* & * & 0 & 0 \\
* & * & * & 0
\end{array}\right), \mathbf{P}^{\prime}=\left(\begin{array}{cccc}
0 & a_{33} x_{3} & a_{33} x_{3} & 0 \\
* & 0 & 0 & a_{64} x_{4} \\
* & * & 0 & 0 \\
* & * & * & 0
\end{array}\right)
$$

Now by means of (4.1) and (4.3), the integrals of motion can be found for this Lie group as follows:

$$
H_{1}=\frac{a_{33} x_{3}}{p_{13}}+\frac{a_{64} x_{4}}{p_{24}}, H_{2}=\frac{1}{2}\left(\left(\frac{a_{33} x_{3}}{p_{13}}\right)^{2}+\left(\frac{a_{64} x_{4}}{p_{24}}\right)^{2}\right) .
$$

\section{Lie group II $\oplus \mathbf{R}$ :}

Inserting $P$ and $P^{\prime}$ in (2.12) one can obtain the compatible Poisson structures $\mathbf{P}$ and $\mathbf{P}^{\prime}$ on the Lie 
group $\mathbf{I I} \oplus \mathbf{R}$ as follows:

$$
\mathbf{P}=\left(\begin{array}{cccc}
0 & p_{12} & p_{13} & 0 \\
* & 0 & 0 & 0 \\
* & * & 0 & p_{34} \\
* & * & * & 0
\end{array}\right), \mathbf{P}^{\prime}=\left(\begin{array}{ccccc}
0 & a_{44} x_{2} & p_{13}^{\prime}+a_{32} x_{2}-a_{44} x_{3} & a_{44} x_{4} \\
* & 0 & p_{23}^{\prime} & 0 \\
* & * & 0 & 0 \\
* & * & * & 0
\end{array}\right)
$$

Now by means of (4.1) and (4.3), the integrals of motion can be found for this Lie group as follows:

$$
H_{1}=\frac{a_{44} x_{2}}{p_{12}}, H_{2}=\frac{a_{44}\left(a_{44} p_{34} x_{2}^{2}-2 p_{12} p_{23}^{\prime} x_{4}\right)}{2 p_{12}^{2} p_{34}} .
$$

\section{Some compatible Poisson structures on symplectic nilpotent six dimensional real Lie algebras}

In this section, we will solve matrix equations (2.17), (2.21) and (2.22) for symplectic nilpotent six dimensional real Lie groups in order to obtain $P$ and $P^{\prime}$. The list of symplectic real six dimensional nilpotent Lie algebras is given in appendix B. Note that for calculating $P$ and $P^{\prime}$ from (2.21) and (2.22) we must first calculate vielbeins $e_{\alpha}^{\mu}$ for nilpotent 6-dimensional real Lie groups. To this end, we use the following relation:

$$
g^{-1} d g=e_{\alpha}^{\mu} X_{\mu} d x^{\alpha} \quad g \in \mathbf{G}
$$

With the following parameterizations for the real 6-dimensional Lie groups $\mathbf{G}$ :

$$
g=e^{x_{1} X_{1}} e^{x_{2} X_{2}} e^{x_{3} X_{3}} e^{x_{4} X_{4}} e^{x_{5} X_{5}} e^{x_{6} X_{6}} .
$$

where $X_{i}$ and $x_{i}$ are generators and coordinates of Lie group, respectively. Then, for left invariant Lie algebra valued one forms, we have:

$$
\begin{aligned}
g^{-1} d g= & d x_{1} e^{-x_{6} X_{6}} e^{-x_{5} X_{5}} e^{-x_{4} X_{4}} e^{-x_{3} X_{3}}\left(e^{-x_{2} X_{2}} X_{1} e^{x_{2} X_{2}}\right) e^{x_{3} X_{3}} e^{x_{4} X_{4}} e^{x_{5} X_{5}} e^{x_{6} X_{6}} \\
& +d x_{2} e^{-x_{6} X_{6}} e^{-x_{5} X_{5}} e^{-x_{4} X_{4}}\left(e^{-x_{3} X_{3}} X_{2} e^{x_{3} X_{3}}\right) e^{x_{4} X_{4}} e^{x_{5} X_{5}} e^{x_{6} X_{6}} \\
& +d x_{3} e^{-x_{6} X_{6}} e^{-x_{5} X_{5}}\left(e^{-x_{4} X_{4}} X_{3} e^{x_{4} X_{4}}\right) e^{x_{5} X_{5}} e^{x_{6} X_{6}} \\
& +d x_{4} e^{-x_{6} X_{6}}\left(e^{-x_{5} X_{5}} X_{4} e^{x_{5} X_{5}}\right) e^{x_{6} X_{6}}+d x_{5} e^{-x_{6} X_{6}} X_{5} e^{x_{6} X_{6}}+d x_{6} X_{6}
\end{aligned}
$$

such that, for this calculation one can use the following relation [7]

$$
\left(e^{-x_{i} X_{i}} X_{j} e^{x_{i} X_{i}}\right)=\left(e^{x_{i} \mathscr{X}_{i}}\right)_{j}^{k} X_{k}
$$

in which we have a summation over the index $k$ on the right hand side but there is not summation over the index $i$. In this way one can calculate all left invariant one forms and vielbeins.

Let us consider an example for calculating of $P$ and $P^{\prime}$; for Lie algebra $A_{6,1}$ we have the following non zero commutators and the matrices $\mathscr{X}_{i}$ and $\mathscr{Y}^{i}$ :

$$
\left[\mathbf{e}_{1}, \mathbf{e}_{2}\right]=\mathbf{e}_{3},\left[\mathbf{e}_{1}, \mathbf{e}_{3}\right]=\mathbf{e}_{4},\left[\mathbf{e}_{1}, \mathbf{e}_{5}\right]=\mathbf{e}_{6},
$$




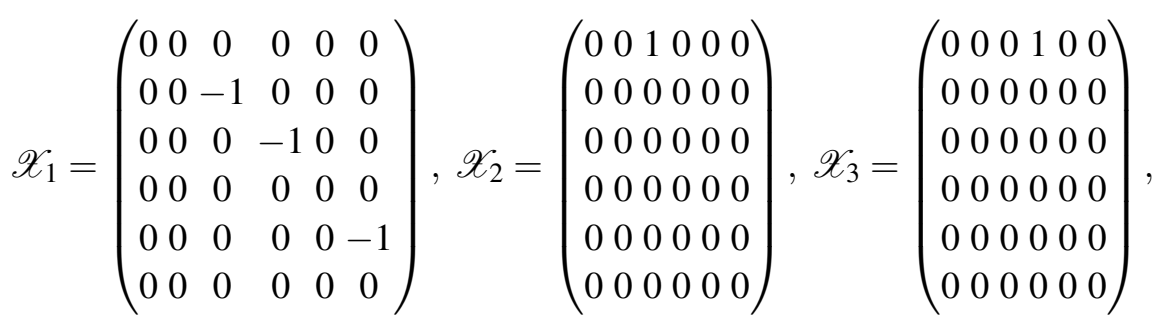

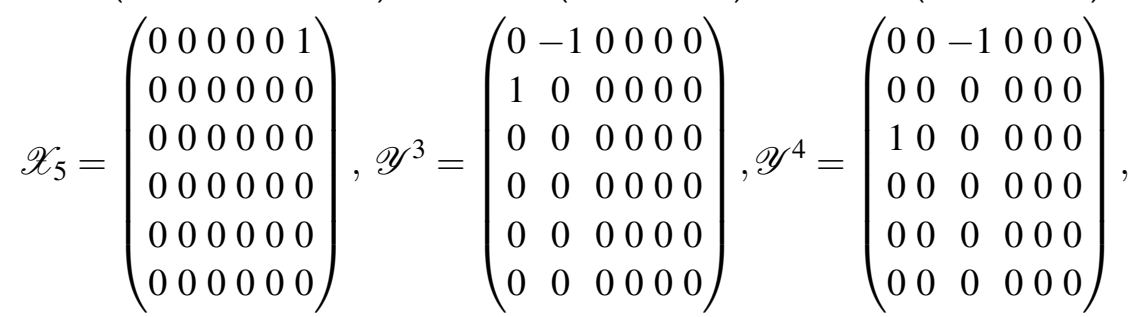

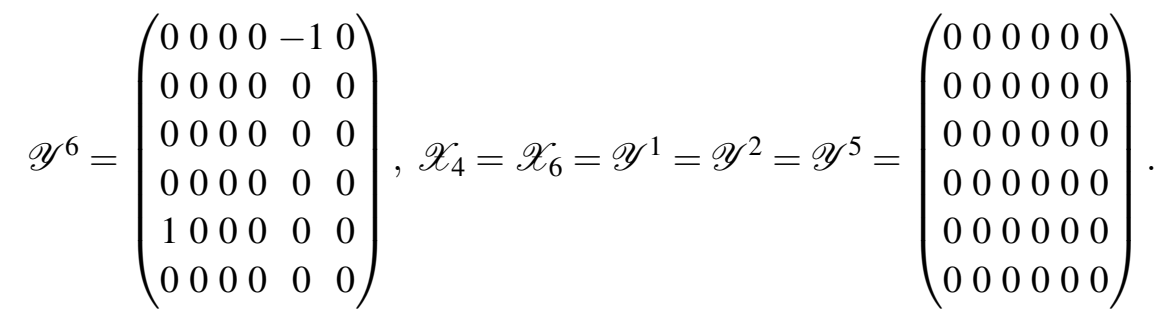

For the Lie algebra $A_{6,1}$ the vielbein matrix $e_{\alpha}^{\mu}$ has the following form:

$$
\left(e_{\alpha}^{\mu}\right)=\left(\begin{array}{cccccc}
1 & 0 & x_{2} & x_{3} & 0 & x_{5} \\
0 & 1 & 0 & 0 & 0 & 0 \\
0 & 0 & 1 & 0 & 0 & 0 \\
0 & 0 & 0 & 1 & 0 & 0 \\
0 & 0 & 0 & 0 & 1 & 0 \\
0 & 0 & 0 & 0 & 0 & 1
\end{array}\right)
$$

Now, substituting $\mathscr{X}_{i}, \mathscr{Y}^{i}$ and $e_{\alpha}^{\mu}$ in (2.17), (2.21) and (2.22) one can obtain the compatible Poisson structures $P$ and $P^{\prime}$ for Lie algebra $A_{6,1}$. One of the solutions has the following forms:

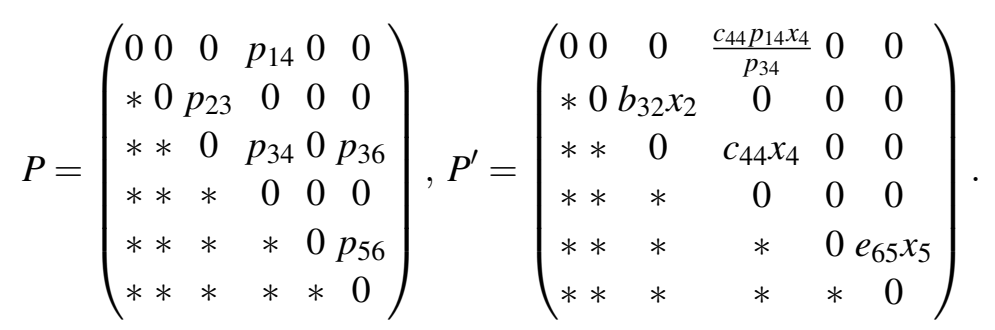

In this way we have obtained some compatible Poisson structures ( $P$ is constant and $P^{\prime}$ as linear function of Lie group coordinates) on symplectic nilpotent six dimensional real Lie algebras, the results are summarized in Table 2. 
J. Abedi-Fardad et al. / Compatible Poisson structures and integrable bi-Hamiltonian systems

Table 2: Compatible Poisson structures on nilpotent 6-dimensional real Lie algebra.

\begin{tabular}{|c|c|c|c|}
\hline g & & Non-zero Poisson structure relations & Comments \\
\hline$A_{6,1}$ & $\begin{array}{l}P \\
P^{\prime}\end{array}$ & $\begin{array}{l}\left\{x_{1}, x_{4}\right\}=p_{14},\left\{x_{2}, x_{3}\right\}=p_{23},\left\{x_{3}, x_{4}\right\}=p_{34},\left\{x_{3}, x_{4}\right\}=p_{36},\left\{x_{5}, x_{6}\right\}=p_{56} \\
\left\{x_{1}, x_{4}\right\}=\frac{c_{44} p_{14} x_{4}}{p_{34}},\left\{x_{2}, x_{3}\right\}=b_{32} x_{2},\left\{x_{3}, x_{4}\right\}=c_{44} x_{4},\left\{x_{5}, x_{6}\right\}=e_{65} x_{5}\end{array}$ & $p_{14} p_{26} \neq 0$ \\
\hline$A_{6,7}$ & $\begin{array}{l}P \\
P^{\prime}\end{array}$ & $\begin{array}{l}\left\{x_{1}, x_{5}\right\}=p_{15},\left\{x_{2}, x_{6}\right\}=p_{26},\left\{x_{3}, x_{4}\right\}=p_{34},\left\{x_{4}, x_{5}\right\}=p_{45} \\
\left\{x_{1}, x_{5}\right\}=\frac{a_{51} x_{1}}{p_{34}},\left\{x_{2}, x_{6}\right\}=b_{62} x_{2}+b_{66} x_{6},\left\{x_{3}, x_{4}\right\}=c_{43} x_{3}\end{array}$ & $p_{15} p_{26} p_{34} \neq 0$ \\
\hline$A_{6,9}$ & $P$ & $\begin{array}{l}\left\{x_{1}, x_{4}\right\}=p_{14},\left\{x_{2}, x_{6}\right\}=-p_{35},\left\{x_{3}, x_{5}\right\}=p_{35},\left\{x_{3}, x_{6}\right\}=p_{36},\left\{x_{4}, x_{6}\right\}=p_{46} \\
\left\{x_{5}, x_{6}\right\}=p_{56} \\
\left\{x_{1}, x_{4}\right\}=a_{41} x_{1}+a_{44} x_{4}+\frac{a_{44} p_{46} x_{2}}{p_{35}},\left\{x_{2}, x_{6}\right\}=-\frac{d_{62} p_{35} x_{2}}{p_{46}} \\
\left\{x_{3}, x_{5}\right\}=\frac{d_{62} p_{35} x_{2}}{p_{46}},\left\{x_{3}, x_{6}\right\}=c_{62} x_{2}+c_{65} x_{5},\left\{x_{4}, x_{6}\right\}=d_{62} x_{2},\left\{x_{5}, x_{6}\right\}=e_{62} x_{2}-\frac{d_{62} p_{35} x_{5}}{p_{46}}\end{array}$ & $p_{14} p_{35} \neq 0$ \\
\hline$A_{6,24}$ & $\begin{array}{l}P \\
P^{\prime}\end{array}$ & $\begin{array}{l}\left\{x_{1}, x_{3}\right\}=p_{13},\left\{x_{2}, x_{5}\right\}=p_{25},\left\{x_{4}, x_{6}\right\}=p_{46} \\
\left\{x_{1}, x_{3}\right\}=a_{31} x_{1}+a_{33} x_{3},\left\{x_{2}, x_{5}\right\}=b_{52} x_{2}+b_{55} x_{5},\left\{x_{4}, x_{6}\right\}=d_{64} x_{4}+d_{66} x_{6}\end{array}$ & $p_{13} p_{25} p_{46} \neq 0$ \\
\hline$A_{6,25}$ & $\begin{array}{l}P \\
P^{\prime}\end{array}$ & $\begin{array}{l}\left\{x_{1}, x_{3}\right\}=p_{13},\left\{x_{2}, x_{4}\right\}=p_{24},\left\{x_{3}, x_{6}\right\}=p_{36},\left\{x_{5}, x_{6}\right\}=p_{56} \\
\left\{x_{1}, x_{3}\right\}=a_{31} x_{1},\left\{x_{2}, x_{4}\right\}=b_{42} x_{2}+b_{44} x_{4},\left\{x_{5}, x_{6}\right\}=e_{65} x_{5}\end{array}$ & $p_{13} p_{24} p_{56} \neq 0$ \\
\hline$A_{6,26}$ & $P^{\prime}$ & $\begin{array}{l}\left\{x_{1}, x_{4}\right\}=p_{14},\left\{x_{2}, x_{3}\right\}=p_{23},\left\{x_{3}, x_{4}\right\}=\frac{c_{44} p_{14}}{a_{44}},\left\{x_{3}, x_{5}\right\}=p_{35},\left\{x_{3}, x_{6}\right\}=\frac{d_{64} p_{35}}{d_{54}}, \\
\left\{x_{4}, x_{5}\right\}=\frac{d_{54} p_{14}}{a_{44}},\left\{x_{4}, x_{6}\right\}=\frac{d_{64} p_{14}}{a_{44}},\left\{x_{5}, x_{6}\right\}=p_{56} \\
\left\{x_{1}, x_{4}\right\}=a_{44} x_{4},\left\{x_{2}, x_{3}\right\}=b_{32} x_{2},\left\{x_{3}, x_{4}\right\}=c_{44} x_{4},\left\{x_{4}, x_{5}\right\}=d_{54} x_{4} \\
\left\{x_{4}, x_{6}\right\}=d_{64} x_{4},\left\{x_{5}, x_{6}\right\}=e_{65} x_{5}-\frac{d_{54} e_{65} x_{6}}{d_{64}}\end{array}$ & $p_{13} p_{24} p_{56} \neq 0$ \\
\hline$A_{6,27}$ & $\begin{array}{l}P \\
P^{\prime}\end{array}$ & $\begin{array}{l}\left\{x_{1}, x_{2}\right\}=p_{12},\left\{x_{1}, x_{3}\right\}=p_{13},\left\{x_{2}, x_{4}\right\}=p_{24},\left\{x_{5}, x_{6}\right\}=p_{56} \\
\left\{x_{1}, x_{3}\right\}=a_{33} x_{3},\left\{x_{2}, x_{4}\right\}=b_{44} x_{4},\left\{x_{5}, x_{6}\right\}=e_{65} x_{5}+e_{66} x_{6}\end{array}$ & $p_{13} p_{24} p_{56} \neq 0$ \\
\hline$A_{6,32}$ & $\begin{array}{l}P \\
P^{\prime}\end{array}$ & $\begin{array}{l}\left\{x_{1}, x_{2}\right\}=p_{12},\left\{x_{1}, x_{4}\right\}=-p_{23},\left\{x_{1}, x_{6}\right\}=\frac{a_{63} p_{23}+a_{25} p_{56}}{b_{33}},\left\{x_{2}, x_{3}\right\}=p_{23},\left\{x_{5}, x_{6}\right\}=p_{56} \\
\left\{x_{1}, x_{2}\right\}=-b_{33} x_{1}-b_{34} x_{2}+a_{23} x_{3}+a_{24} x_{4}+a_{25} x_{5},\left\{x_{1}, x_{4}\right\}=-b_{33} x_{3}-b_{34} x_{4}, \\
\left\{x_{1}, x_{6}\right\}=a_{63} x_{3}+\frac{a_{63}\left(b_{33} b_{34}+a_{25} e_{66}\right) x_{4}}{b_{33}^{2}}+\frac{a_{25} e_{65} x_{5}+a_{25} e_{66} x_{6}}{b_{33}},\left\{x_{2}, x_{3}\right\}=b_{33} x_{3}+b_{34} x_{4}, \\
\left\{x_{5}, x_{6}\right\}=\frac{a_{63} e_{66} x_{4}}{b_{33}}+e_{65} x_{5}+e_{66} x_{6}\end{array}$ & $p_{23} p_{56} \neq 0$ \\
\hline
\end{tabular}

\section{Integrable bi-Hamiltonian systems on symplectic nilpotent six dimensional real Lie groups}

In this section, we construct the integrable bi-Hamiltonian systems with symplectic nilpotent six dimensional real Lie groups as phase space. Using the matrices $P$ and $P^{\prime}$ given in Table 2 and inserting $P$ and $P^{\prime}$ in (2.12) and using the related vielbeins as in appendix B the compatible Poisson structures $\mathbf{P}$ and $\mathbf{P}^{\prime}$ on Lie groups are obtained, then using (4.1) and (4.3) one can find the Hamiltonian and integrals of motions of bi-Hamiltonian systems. In the following we perform this work separately for symplectic nilpotent six dimensional real Lie groups. In this way we obtain new bi-Hamiltonian systems over nilpotent six dimensional real Lie groups as phase spaces.

Lie group $\mathbf{A}_{6,1}$ :

Inserting $P, P^{\prime}$ and vielbeins matrix in (2.12) one can obtain the compatible Poisson structures $\mathbf{P}$ 
and $\mathbf{P}^{\prime}$ on the Lie group $\mathbf{A}_{6,1}$ as follows:

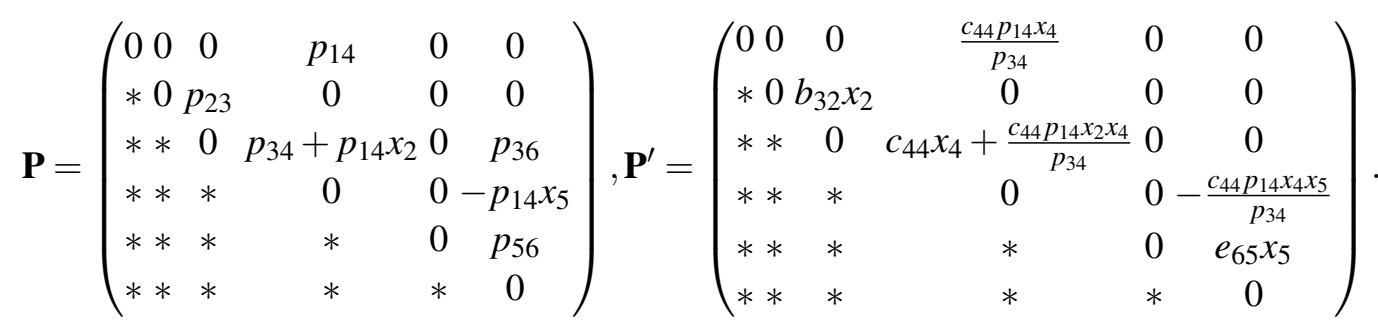

Now by means of (4.1) and (4.3), the integrals of motion can be found for this Lie group as follows:

$$
\begin{gathered}
H_{1}=\frac{b_{32} x_{2}}{p_{23}}+\frac{c_{44} x_{4}}{p_{34}}+\frac{e_{65} x_{5}}{p_{56}}, H_{2}=\frac{1}{2}\left(\left(\frac{b_{32} x_{2}}{p_{23}}\right)^{2}+\left(\frac{c_{44} x_{4}}{p_{34}}\right)^{2}+\left(\frac{e_{65} x_{5}}{p_{56}}\right)^{2}\right), \\
H_{3}=\frac{1}{3}\left(\left(\frac{b_{32} x_{2}}{p_{23}}\right)^{3}+\left(\frac{c_{44} x_{4}}{p_{34}}\right)^{3}+\left(\frac{e_{65} x_{5}}{p_{56}}\right)^{3}\right) .
\end{gathered}
$$

\section{Lie group $\mathbf{A}_{6,7}$ :}

Inserting $P, P^{\prime}$ and vielbeins matrix in (2.12) one can obtain the compatible Poisson structures $\mathbf{P}$ and $\mathbf{P}^{\prime}$ on the Lie group $\mathbf{A}_{6,7}$ as follows:

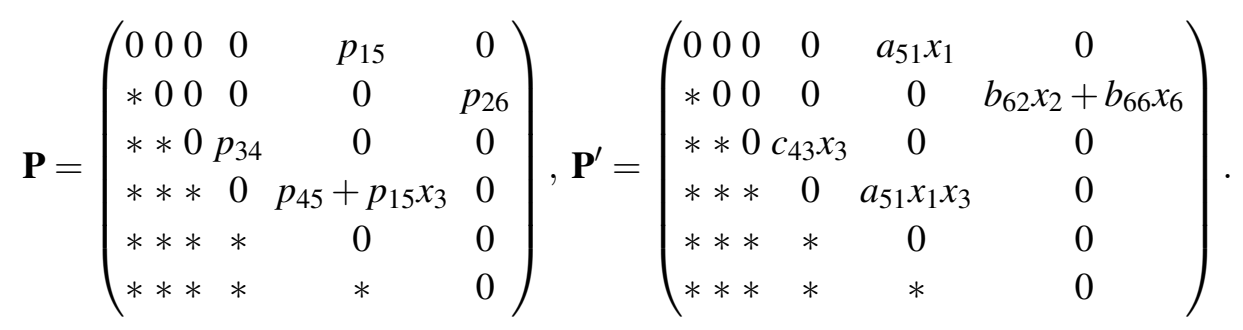

Now by means of (4.3), the integrals of motion can be found for this Lie group as follows:

$$
\begin{gathered}
H_{1}=\frac{a_{51} x_{1}}{p_{15}}+\frac{b_{62} x_{2}}{p_{26}}+\frac{c_{43} x_{3}}{p_{34}}+\frac{b_{66} x_{6}}{p_{26}}, H_{2}=\frac{1}{2}\left(\left(\frac{a_{51} x_{1}}{p_{15}}\right)^{2}+\left(\frac{c_{43} x_{3}}{p_{34}}\right)^{2}+\left(\frac{b_{62} x_{2}+b_{66} x_{6}}{p_{26}}\right)^{2}\right), \\
H_{3}=\frac{1}{3}\left(\left(\frac{a_{51} x_{1}}{p_{15}}\right)^{3}+\left(\frac{c_{43} x_{3}}{p_{34}}\right)^{3}+\left(\frac{b_{62} x_{2}+b_{66} x_{6}}{p_{26}}\right)^{3}\right) .
\end{gathered}
$$

\section{Lie group $\mathbf{A}_{6,9}$ :}

Inserting $P, P^{\prime}$ and vielbeins matrix in (2.12) one can obtain the compatible Poisson structures $\mathbf{P}$ 
and $\mathbf{P}^{\prime}$ on the Lie group $\mathbf{A}_{6,9}$ as follows:

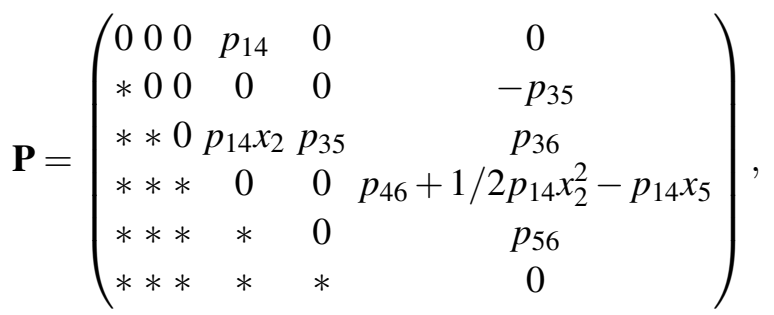

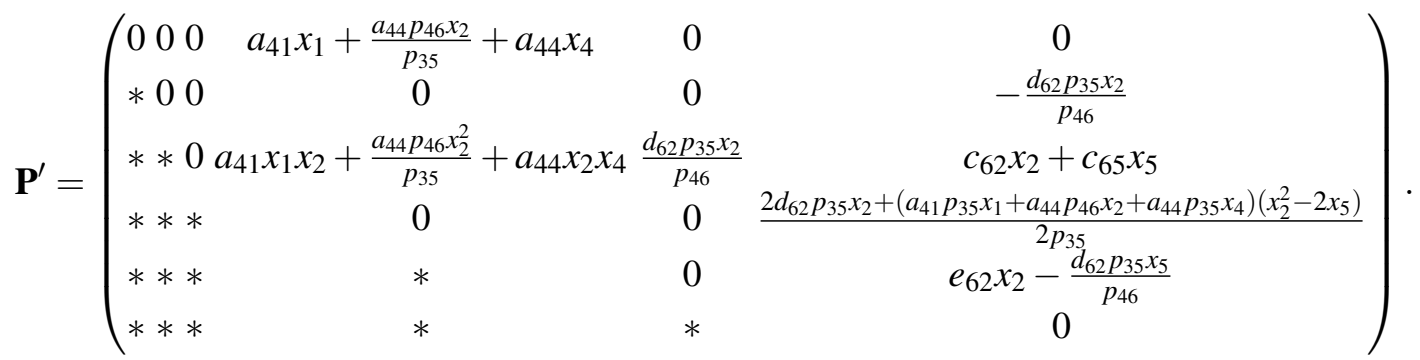

Now by means of (4.3), the integrals of motion can be found for this Lie group as follows:

$$
\begin{aligned}
& H_{1}=\frac{a_{41} p_{35} p_{46} x_{1}+2 d_{62} p_{14} p_{35} x_{2}+a_{44} p_{46}\left(p_{46} x_{2}+p_{35} x_{4}\right)}{p_{14} p_{35} p_{46}} \\
& H_{2}=\frac{1}{4}\left(\left(\frac{4 d_{62} x_{2}}{p_{46}}\right)^{2}+\left(\frac{a_{41} p_{35} x_{1}+a_{44} p_{46} x_{2}+a_{44} p_{35} x_{4}}{p_{14} p_{35}}\right)^{2}+\left(\frac{a_{41} x_{1}+a_{44}\left(\frac{p_{46} x_{2}}{p_{35}}+x_{4}\right)}{p_{14}}\right)^{2}\right), \\
& H_{3}=\frac{1}{6}\left(\left(\frac{4 d_{62} x_{2}}{p_{46}}\right)^{3}+\left(\frac{a_{41} p_{35} x_{1}+a_{44} p_{46} x_{2}+a_{44} p_{35} x_{4}}{p_{14} p_{35}}\right)^{3}+\left(\frac{a_{41} x_{1}+a_{44}\left(\frac{p_{46} x_{2}}{p_{35}}+x_{4}\right)}{p_{14}}\right)^{3}\right) .
\end{aligned}
$$

Lie group $\mathbf{A}_{6,24}$ :

Inserting $P, P^{\prime}$ and vielbeins matrix in (2.12) one can obtain the compatible Poisson structures $\mathbf{P}$ and $\mathbf{P}^{\prime}$ on the Lie group $\mathbf{A}_{6,24}$ as follows:

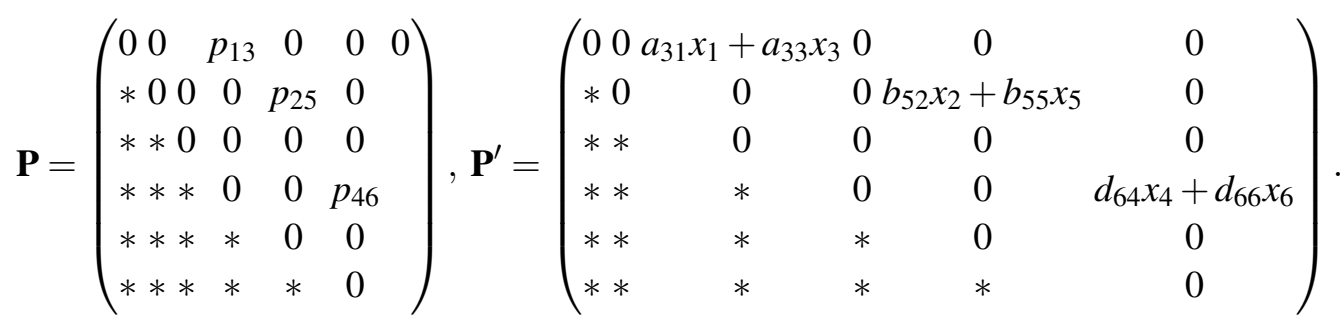

Now by means of (4.3), the integrals of motion can be found for this Lie group as follows:

$$
\begin{aligned}
& H_{1}=\frac{a_{31} x_{1}+a_{33} x_{3}}{p_{13}}+\frac{b_{52} x_{2}+b_{55} x_{5}}{p_{25}}+\frac{d_{64} x_{4}+d_{66} x_{6}}{p_{45}}, \\
& H_{2}=\frac{1}{2}\left(\left(\frac{a_{31} x_{1}+a_{33} x_{3}}{p_{13}}\right)^{2}+\left(\frac{b_{52} x_{2}+b_{55} x_{5}}{p_{25}}\right)^{2}+\left(\frac{d_{64} x_{4}+d_{66} x_{6}}{p_{45}}\right)^{2}\right), \\
& H_{3}=\frac{1}{3}\left(\left(\frac{a_{31} x_{1}+a_{33} x_{3}}{p_{13}}\right)^{3}+\left(\frac{b_{52} x_{2}+b_{55} x_{5}}{p_{25}}\right)^{3}+\left(\frac{d_{64} x_{4}+d_{66} x_{6}}{p_{45}}\right)^{3}\right) .
\end{aligned}
$$

Lie group $\mathbf{A}_{6,25}$ :

Inserting $P, P^{\prime}$ and vielbeins matrix in (2.12) one can obtain the compatible Poisson structures $\mathbf{P}$ 
and $\mathbf{P}^{\prime}$ on the Lie group $\mathbf{A}_{6,25}$ as follows:

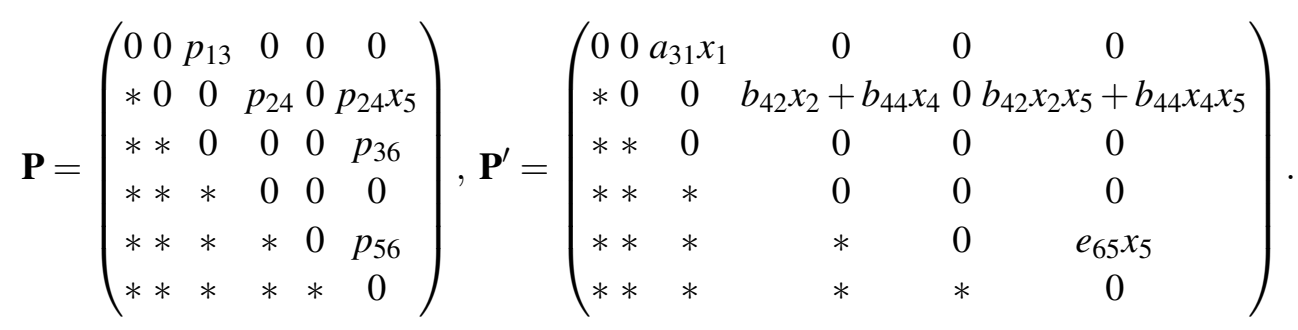

Now by means of (4.3), the integrals of motion can be found for this Lie group as follows:

$$
\begin{gathered}
H_{1}=\frac{a_{31} x_{1}}{p_{13}}+\frac{b_{42} x_{2}+b_{44} x_{4}}{p_{24}}+\frac{e_{65} x_{5}}{p_{56}}, H_{2}=\frac{1}{2}\left(\left(\frac{a_{31} x_{1}}{p_{13}}\right)^{2}+\left(\frac{b_{42} x_{2}+b_{44} x_{4}}{p_{24}}\right)^{2}+\left(\frac{e_{65} x_{5}}{p_{56}}\right)^{2}\right), \\
H_{3}=\frac{1}{3}\left(\left(\frac{a_{31} x_{1}}{p_{13}}\right)^{3}+\left(\frac{b_{42} x_{2}+b_{44} x_{4}}{p_{24}}\right)^{3}+\left(\frac{e_{65} x_{5}}{p_{56}}\right)^{3}\right) .
\end{gathered}
$$

\section{Lie group $\mathbf{A}_{6,26}$ :}

Inserting $P, P^{\prime}$ and vielbeins matrix in (2.12) one can obtain the compatible Poisson structures $\mathbf{P}$ and $\mathbf{P}^{\prime}$ on the Lie group $\mathbf{A}_{6,26}$ as follows:

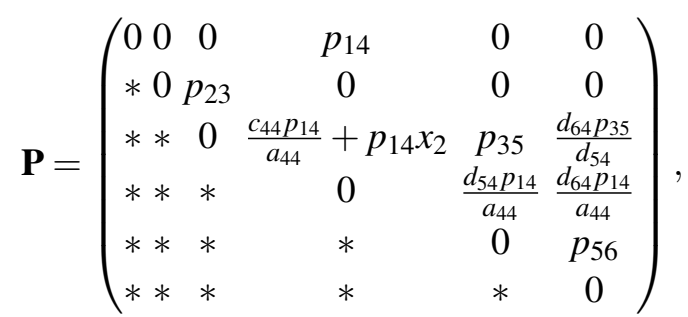

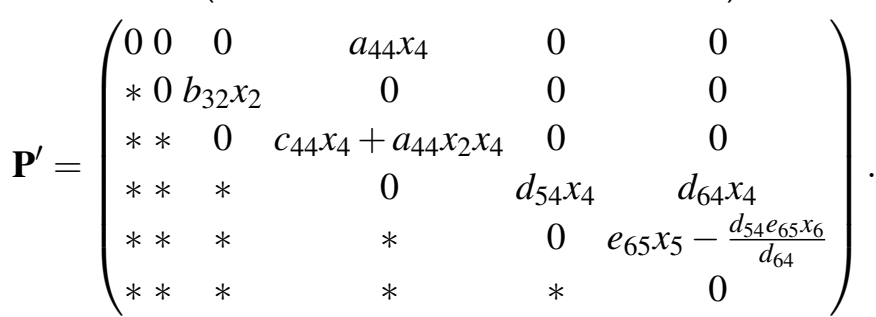

Now by means of (4.3), the integrals of motion can be found for this Lie group as follows:

$$
\begin{gathered}
H_{1}=\frac{b_{32} x_{2}}{p_{23}}+\frac{a_{44} x_{4}}{p_{14}}+\frac{e_{65}\left(d_{64} x_{5}-d_{54} x_{6}\right)}{d_{64} p_{56}}, H_{2}=\frac{1}{2}\left(\left(\frac{b_{32} x_{2}}{p_{23}}\right)^{2}+\left(\frac{a_{44} x_{4}}{p_{14}}\right)^{2}+\left(\frac{e_{65}\left(d_{64} x_{5}-d_{54} x_{6}\right)}{d_{64} p_{56}}\right)^{2}\right), \\
H_{3}=\frac{1}{3}\left(\left(\frac{b_{32} x_{2}}{p_{23}}\right)^{3}+\left(\frac{a_{44} x_{4}}{p_{14}}\right)^{3}+\left(\frac{e_{65}\left(d_{64} x_{5}-d_{54} x_{6}\right)}{d_{64} p_{56}}\right)^{3}\right) .
\end{gathered}
$$

\section{Lie group $\mathbf{A}_{6,27}$ :}

Inserting $P, P^{\prime}$ and vielbeins matrix in (2.12) one can obtain the compatible Poisson structures $\mathbf{P}$ 
and $\mathbf{P}^{\prime}$ on the Lie group $\mathbf{A}_{6,27}$ as follows:

$$
\mathbf{P}=\left(\begin{array}{cccccc}
0 & p_{12} & p_{13} & 0 & 0 & 0 \\
* & 0 & 0 & p_{24} & 0 & 0 \\
* & * & 0 & 0 & 0 & 0 \\
* & * & * & 0 & 0 & 0 \\
* & * & * & * & 0 & p_{56} \\
* & * & * & * & * & 0
\end{array}\right), \mathbf{P}^{\prime}=\left(\begin{array}{cccccc}
0 & 0 & a_{33} x_{3} & 0 & 0 & 0 \\
* 0 & 0 & b_{44} x_{4} & 0 & 0 \\
* * & 0 & 0 & 0 & 0 \\
* * & * & 0 & 0 & \\
* * & * & * & 0 & e_{65} x_{5}+e_{66} x_{6} \\
* * & * & * & * & 0
\end{array}\right) .
$$

Now by means of (4.3), the integrals of motion can be found for this Lie group as follows:

$$
\begin{gathered}
H_{1}=\frac{a_{33} x_{3}}{p_{13}}+\frac{b_{44} x_{4}}{p_{24}}+\frac{e_{65} x_{5}+e_{66} x_{6}}{p_{56}}, H_{2}=\frac{1}{2}\left(\left(\frac{a_{33} x_{3}}{p_{13}}\right)^{2}+\left(\frac{b_{44} x_{4}}{p_{24}}\right)^{2}+\left(\frac{e_{65} x_{5}+e_{66} x_{6}{ }^{2}}{p_{56}}\right)\right), \\
H_{3}=\frac{1}{3}\left(\left(\frac{a_{33} x_{3}}{p_{13}}\right)^{3}+\left(\frac{b_{44} x_{4}}{p_{24}}\right)^{3}+\left(\frac{e_{65} x_{5}+e_{66} x_{6}}{p_{56}}\right)^{3}\right) .
\end{gathered}
$$

\section{Concluding remarks}

Using a procedure based on the adjoint representation of the Lie algebra we give a method for calculation of compatible Poisson structures on four and nilpotent six dimensional symplectic real Lie algebras. Also by use of Magri-Morosi's theorem we have obtained new bi-Hamiltonian systems with these Lie groups as phase spaces. It seems that using of the adjoint representation of the Lie algebra (mainly for low dimensions) is simpler than the Lax-pair method (at least in computation). As an open problem, one can obtain another set of compatible Poisson structures by setting $P^{\prime}$ as a second order functions of the Lie group parameters and in this way obtain new bi-Hamiltonian

\begin{tabular}{|c|c|c|c|}
\hline $\mathbf{g}$ & Non-zero commutation relations & $\mathbf{g}$ & Non-zero commutation relations \\
\hline$A_{4,1}$ & {$\left[\mathbf{e}_{2}, \mathbf{e}_{4}\right]=\mathbf{e}_{1},\left[\mathbf{e}_{3}, \mathbf{e}_{4}\right]=\mathbf{e}_{2}$} & $A_{4,2}^{-1}$ & {$\left[\mathbf{e}_{1}, \mathbf{e}_{4}\right]=-\mathbf{e}_{1},\left[\mathbf{e}_{2}, \mathbf{e}_{4}\right]=\mathbf{e}_{2},\left[\mathbf{e}_{3}, \mathbf{e}_{4}\right]=\mathbf{e}_{2}+\mathbf{e}_{3}$} \\
\hline$A_{4,3}$ & {$\left[\mathbf{e}_{1}, \mathbf{e}_{4}\right]=\mathbf{e}_{1},\left[\mathbf{e}_{3}, \mathbf{e}_{4}\right]=\mathbf{e}_{2}$} & $A_{4,5}^{-1,-1}$ & {$\left[\mathbf{e}_{1}, \mathbf{e}_{4}\right]=\mathbf{e}_{1},\left[\mathbf{e}_{2}, \mathbf{e}_{4}\right]=-\mathbf{e}_{2},\left[\mathbf{e}_{3}, \mathbf{e}_{4}\right]=-\mathbf{e}_{3}$} \\
\hline$A_{4,5}^{-1, b}$ & {$\left[\mathbf{e}_{1}, \mathbf{e}_{4}\right]=\mathbf{e}_{1},\left[\mathbf{e}_{2}, \mathbf{e}_{4}\right]=-\mathbf{e}_{2},\left[\mathbf{e}_{3}, \mathbf{e}_{4}\right]=b \mathbf{e}_{3}$} & $A_{4,5}^{a,-1}$ & {$\left[\mathbf{e}_{1}, \mathbf{e}_{4}\right]=\mathbf{e}_{1},\left[\mathbf{e}_{2}, \mathbf{e}_{4}\right]=a \mathbf{e}_{2},\left[\mathbf{e}_{3}, \mathbf{e}_{4}\right]=-\mathbf{e}_{3}$} \\
\hline$A_{4,5}^{a,-a}$ & {$\left[\mathbf{e}_{1}, \mathbf{e}_{4}\right]=\mathbf{e}_{1},\left[\mathbf{e}_{2}, \mathbf{e}_{4}\right]=a \mathbf{e}_{2},\left[\mathbf{e}_{3}, \mathbf{e}_{4}\right]=-a \mathbf{e}_{3}$} & $A_{4,6}^{a, 0}$ & {$\left[\mathbf{e}_{1}, \mathbf{e}_{4}\right]=a \mathbf{e}_{1}, \quad\left[\mathbf{e}_{2}, \mathbf{e}_{4}\right]=-\mathbf{e}_{3},\left[\mathbf{e}_{3}, \mathbf{e}_{4}\right]=\mathbf{e}_{2}$} \\
\hline$A_{4,7}$ & $\begin{aligned} {\left[\mathbf{e}_{1}, \mathbf{e}_{4}\right] } & =2 \mathbf{e}_{1},\left[\mathbf{e}_{2}, \mathbf{e}_{3}\right]=\mathbf{e}_{1},\left[\mathbf{e}_{2}, \mathbf{e}_{4}\right]=\mathbf{e}_{2} \\
{\left[\mathbf{e}_{3}, \mathbf{e}_{4}\right] } & =\mathbf{e}_{2}+\mathbf{e}_{3}\end{aligned}$ & $\begin{array}{l}A_{4,9}^{0} \\
A_{4,9}^{-1 / 2}\end{array}$ & $\begin{array}{l}{\left[\mathbf{e}_{1}, \mathbf{e}_{4}\right]=\mathbf{e}_{1},\left[\mathbf{e}_{2}, \mathbf{e}_{3}\right]=\mathbf{e}_{1},\left[\mathbf{e}_{2}, \mathbf{e}_{4}\right]=\mathbf{e}_{2}} \\
{\left[\mathbf{e}_{1}, \mathbf{e}_{4}\right]=1 / 2 \mathbf{e}_{1},\left[\mathbf{e}_{2}, \mathbf{e}_{3}\right]=\mathbf{e}_{1},\left[\mathbf{e}_{2}, \mathbf{e}_{4}\right]=\mathbf{e}_{2}}\end{array}$ \\
\hline$A_{4,9}^{1}$ & {$\left[\mathbf{e}_{1}, \mathbf{e}_{4}\right]=2 \mathbf{e}_{1},\left[\mathbf{e}_{2}, \mathbf{e}_{3}\right]=\mathbf{e}_{1},\left[\mathbf{e}_{2}, \mathbf{e}_{4}\right]=\mathbf{e}_{2}$} & & {$\left[\mathbf{e}_{3}, \mathbf{e}_{4}\right]=-1 / 2 \mathbf{e}_{3}$} \\
\hline & {$\left[\mathbf{e}_{3}, \mathbf{e}_{4}\right]=\mathbf{e}_{3}$} & $A_{4,9}^{b}$ & {$\left[\mathbf{e}_{1}, \mathbf{e}_{4}\right]=(1+b) \mathbf{e}_{1},\left[\mathbf{e}_{2}, \mathbf{e}_{3}\right]=\mathbf{e}_{1},\left[\mathbf{e}_{2}, \mathbf{e}_{4}\right]=\mathbf{e}_{2}$} \\
\hline$A_{4,11}^{b}$ & {$\left[\mathbf{e}_{1}, \mathbf{e}_{4}\right]=2 b \mathbf{e}_{1},\left[\mathbf{e}_{2}, \mathbf{e}_{3}\right]=\mathbf{e}_{1}$} & & {$\left[\mathbf{e}_{3}, \mathbf{e}_{4}\right]=b \mathbf{e}_{3}$} \\
\hline & {$\left[\mathbf{e}_{2}, \mathbf{e}_{4}\right]=b \mathbf{e}_{2}-\mathbf{e}_{3},\left[\mathbf{e}_{3}, \mathbf{e}_{4}\right]=\mathbf{e}_{2}+b \mathbf{e}_{3}$} & $A_{4,12}$ & {$\left[\mathbf{e}_{1}, \mathbf{e}_{3}\right]=\mathbf{e}_{1},\left[\mathbf{e}_{1}, \mathbf{e}_{4}\right]=-\mathbf{e}_{2},\left[\mathbf{e}_{2}, \mathbf{e}_{3}\right]=\mathbf{e}_{2}$} \\
\hline$A_{2} \oplus A_{2}$ & {$\left[\mathbf{e}_{1}, \mathbf{e}_{2}\right]=\mathbf{e}_{2},\left[\mathbf{e}_{3}, \mathbf{e}_{4}\right]=\mathbf{e}_{4}$} & & {$\left[\mathbf{e}_{2}, \mathbf{e}_{4}\right]=\mathbf{e}_{1}$} \\
\hline$V I_{0} \oplus R$ & {$\left[\mathbf{e}_{1}, \mathbf{e}_{3}\right]=\mathbf{e}_{2},\left[\mathbf{e}_{2}, \mathbf{e}_{3}\right]=\mathbf{e}_{1}$} & $I I I \oplus R$ & {$\left[\mathbf{e}_{1}, \mathbf{e}_{2}\right]=-\mathbf{e}_{2}-\mathbf{e}_{3},\left[\mathbf{e}_{1}, \mathbf{e}_{3}\right]=-\mathbf{e}_{2}-\mathbf{e}_{3}$} \\
\hline$V I I_{0} \oplus R$ & {$\left[\mathbf{e}_{1}, \mathbf{e}_{3}\right]=-\mathbf{e}_{2},\left[\mathbf{e}_{2}, \mathbf{e}_{3}\right]=\mathbf{e}_{1}$} & $I I \oplus R$ & {$\left[\mathbf{e}_{2}, \mathbf{e}_{3}\right]=\mathbf{e}_{1}$} \\
\hline
\end{tabular}
systems.

Appendix A: The list of symplectic four dimensional real Lie algebras [1] 
Appendix B: The list of symplectic nilpotent 6-dimensional real Lie algebras and vielbeins matrix

\begin{tabular}{|c|c|c|}
\hline g & Non-zero commutation relations & vielbeins matrix \\
\hline$A_{6,1}$ & $\begin{array}{l}{\left[\mathbf{e}_{1}, \mathbf{e}_{2}\right]=\mathbf{e}_{3}} \\
{\left[\mathbf{e}_{1}, \mathbf{e}_{3}\right]=\mathbf{e}_{4}} \\
{\left[\mathbf{e}_{1}, \mathbf{e}_{5}\right]=\mathbf{e}_{6}}\end{array}$ & $\begin{array}{l}\left(\begin{array}{cccccc}1 & 0 & x_{2} & x_{3} & 0 & x_{5} \\
0 & 1 & 0 & 0 & 0 & 0 \\
0 & 0 & 1 & 0 & 0 & 0 \\
0 & 0 & 0 & 1 & 0 & 0 \\
0 & 0 & 0 & 0 & 1 & 0 \\
0 & 0 & 0 & 0 & 0 & 1\end{array}\right) \\
\left(\begin{array}{llllll}1 & 0 & x_{2} & x_{3} & x_{4} & x_{5}\end{array}\right)\end{array}$ \\
\hline$A_{6,2}$ & $\begin{array}{l}{\left[\mathbf{e}_{1}, \mathbf{e}_{2}\right]=\mathbf{e}_{3}} \\
{\left[\mathbf{e}_{1}, \mathbf{e}_{3}\right]=\mathbf{e}_{4}} \\
{\left[\mathbf{e}_{1}, \mathbf{e}_{4}\right]=\mathbf{e}_{5}} \\
{\left[\mathbf{e}_{1}, \mathbf{e}_{5}\right]=\mathbf{e}_{6}}\end{array}$ & $\left.\begin{array}{llllll}0 & 1 & 0 & 0 & 0 & 0 \\
0 & 0 & 1 & 0 & 0 & 0 \\
0 & 0 & 0 & 1 & 0 & 0 \\
0 & 0 & 0 & 0 & 1 & 0 \\
0 & 0 & 0 & 0 & 0 & 1\end{array}\right)$ \\
\hline$A_{6,3}$ & $\begin{array}{l}{\left[\mathbf{e}_{1}, \mathbf{e}_{2}\right]=\mathbf{e}_{6}} \\
{\left[\mathbf{e}_{1}, \mathbf{e}_{3}\right]=\mathbf{e}_{4}} \\
{\left[\mathbf{e}_{2}, \mathbf{e}_{3}\right]=\mathbf{e}_{5}}\end{array}$ & $\left(\begin{array}{cccccc}1 & 0 & 0 & x_{3} & 0 & x_{2} \\
0 & 1 & 0 & 0 & x_{3} & 0 \\
0 & 0 & 1 & 0 & 0 & 0 \\
0 & 0 & 0 & 1 & 0 & 0 \\
0 & 0 & 0 & 0 & 1 & 0 \\
0 & 0 & 0 & 0 & 0 & 1\end{array}\right)^{\prime}$ \\
\hline$A_{6,4}$ & $\begin{array}{l}{\left[\mathbf{e}_{1}, \mathbf{e}_{2}\right]=\mathbf{e}_{5}} \\
{\left[\mathbf{e}_{1}, \mathbf{e}_{3}\right]=\mathbf{e}_{6}} \\
{\left[\mathbf{e}_{2}, \mathbf{e}_{4}\right]=\mathbf{e}_{6}}\end{array}$ & $\left(\begin{array}{cccccc}1 & 0 & 0 & 0 & x_{2} & x_{3} \\
0 & 1 & 0 & 0 & 0 & x_{4} \\
0 & 0 & 1 & 0 & 0 & 0 \\
0 & 0 & 0 & 1 & 0 & 0 \\
0 & 0 & 0 & 0 & 1 & 0 \\
0 & 0 & 0 & 0 & 0 & 1\end{array}\right)$ \\
\hline$A_{6,5}$ & $\begin{array}{l}{\left[\mathbf{e}_{1}, \mathbf{e}_{3}\right]=\mathbf{e}_{5}} \\
{\left[\mathbf{e}_{1}, \mathbf{e}_{4}\right]=\mathbf{e}_{6}} \\
{\left[\mathbf{e}_{2}, \mathbf{e}_{3}\right]=a \mathbf{e}_{6}} \\
{\left[\mathbf{e}_{2}, \mathbf{e}_{4}\right]=\mathbf{e}_{5}} \\
\quad a= \pm 1\end{array}$ & $\left(\begin{array}{cccccc}1 & 0 & 0 & 0 & x_{3} & x_{4} \\
0 & 1 & 0 & 0 & x_{4} & a x_{3} \\
0 & 0 & 1 & 0 & 0 & 0 \\
0 & 0 & 0 & 1 & 0 & 0 \\
0 & 0 & 0 & 0 & 1 & 0 \\
0 & 0 & 0 & 0 & 0 & 1 \\
(1 & 0 & 0 & x_{3} & x_{4} & x_{5}\end{array}\right)$ \\
\hline$A_{6,6}$ & $\begin{array}{l}{\left[\mathbf{e}_{1}, \mathbf{e}_{2}\right]=\mathbf{e}_{6}} \\
{\left[\mathbf{e}_{1}, \mathbf{e}_{3}\right]=\mathbf{e}_{4}} \\
{\left[\mathbf{e}_{1}, \mathbf{e}_{4}\right]=\mathbf{e}_{5}} \\
{\left[\mathbf{e}_{2}, \mathbf{e}_{3}\right]=\mathbf{e}_{5}}\end{array}$ & $\left.\begin{array}{cccccc}0 & 1 & 0 & 0 & x_{3} & 0 \\
0 & 0 & 1 & 0 & 0 & 0 \\
0 & 0 & 0 & 1 & 0 & 0 \\
0 & 0 & 0 & 0 & 1 & 0 \\
0 & 0 & 0 & 0 & 0 & 1\end{array}\right)$ \\
\hline$A_{6,7}$ & $\begin{array}{l}{\left[\mathbf{e}_{1}, \mathbf{e}_{3}\right]=\mathbf{e}_{4}} \\
{\left[\mathbf{e}_{1}, \mathbf{e}_{4}\right]=\mathbf{e}_{5}} \\
{\left[\mathbf{e}_{2}, \mathbf{e}_{3}\right]=\mathbf{e}_{6}}\end{array}$ & $\begin{array}{l}\left(\begin{array}{llllll}1 & 0 & 0 & x_{3} & x_{4} & 0 \\
0 & 1 & 0 & 0 & 0 & x_{3} \\
0 & 0 & 1 & 0 & 0 & 0 \\
0 & 0 & 0 & 1 & 0 & 0 \\
0 & 0 & 0 & 0 & 1 & 0 \\
0 & 0 & 0 & 0 & 0 & 1\end{array}\right) \\
\left(\begin{array}{llllll}1 & 0 & x_{2} & x_{3} & x_{2} & -x_{2}^{2} / 2\end{array}\right)\end{array}$ \\
\hline$A_{6,8}$ & $\begin{array}{l}{\left[\mathbf{e}_{1}, \mathbf{e}_{2}\right]=\mathbf{e}_{3}+\mathbf{e}_{5}} \\
{\left[\mathbf{e}_{1}, \mathbf{e}_{3}\right]=\mathbf{e}_{4}} \\
{\left[\mathbf{e}_{2}, \mathbf{e}_{5}\right]=\mathbf{e}_{6}}\end{array}$ & $\begin{array}{cccccc}0 & 1 & 0 & 0 & 0 & x 5 \\
0 & 0 & 1 & 0 & 0 & 0 \\
0 & 0 & 0 & 1 & 0 & 0 \\
0 & 0 & 0 & 0 & 1 & 0 \\
0 & 0 & 0 & 0 & 0 & 1\end{array}$ \\
\hline
\end{tabular}




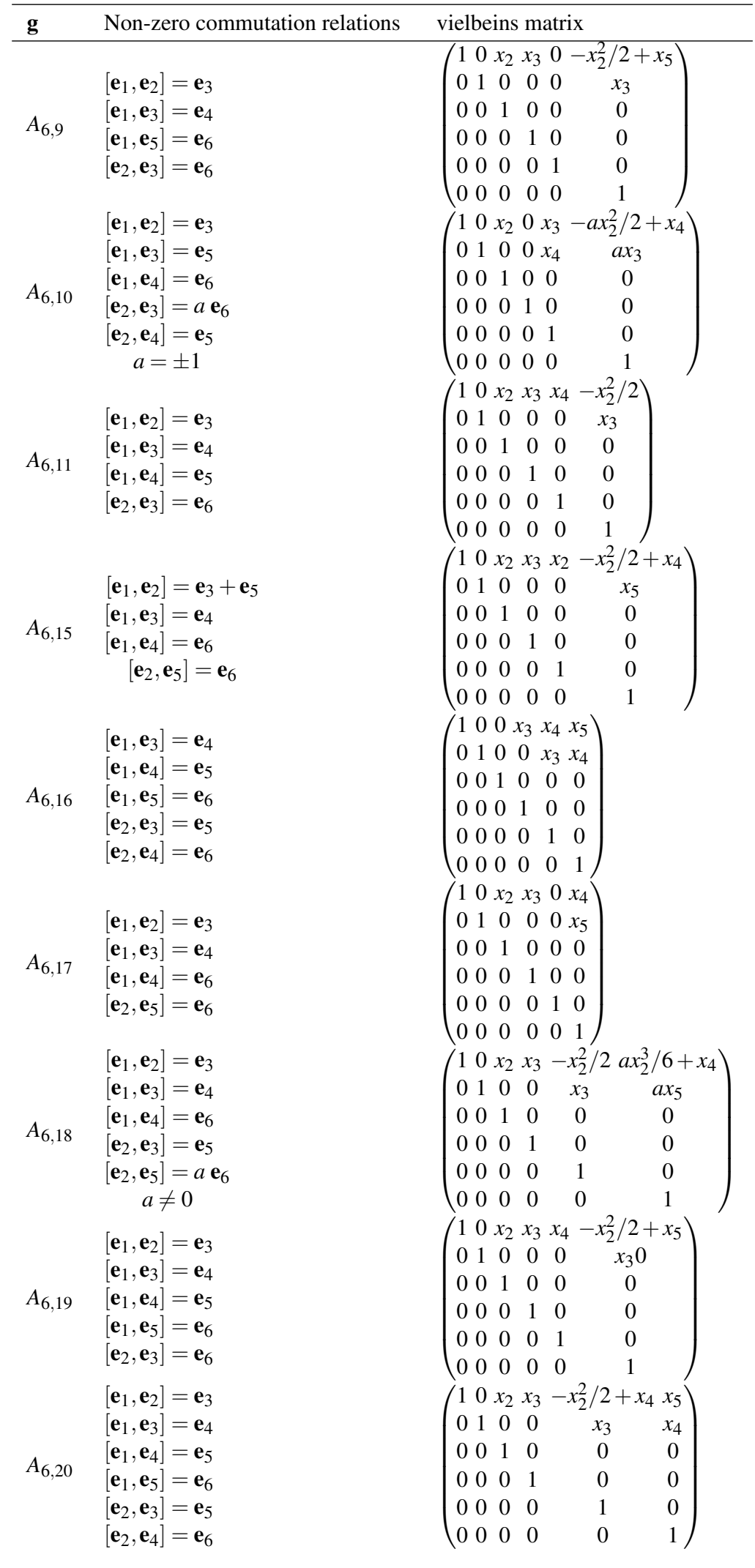




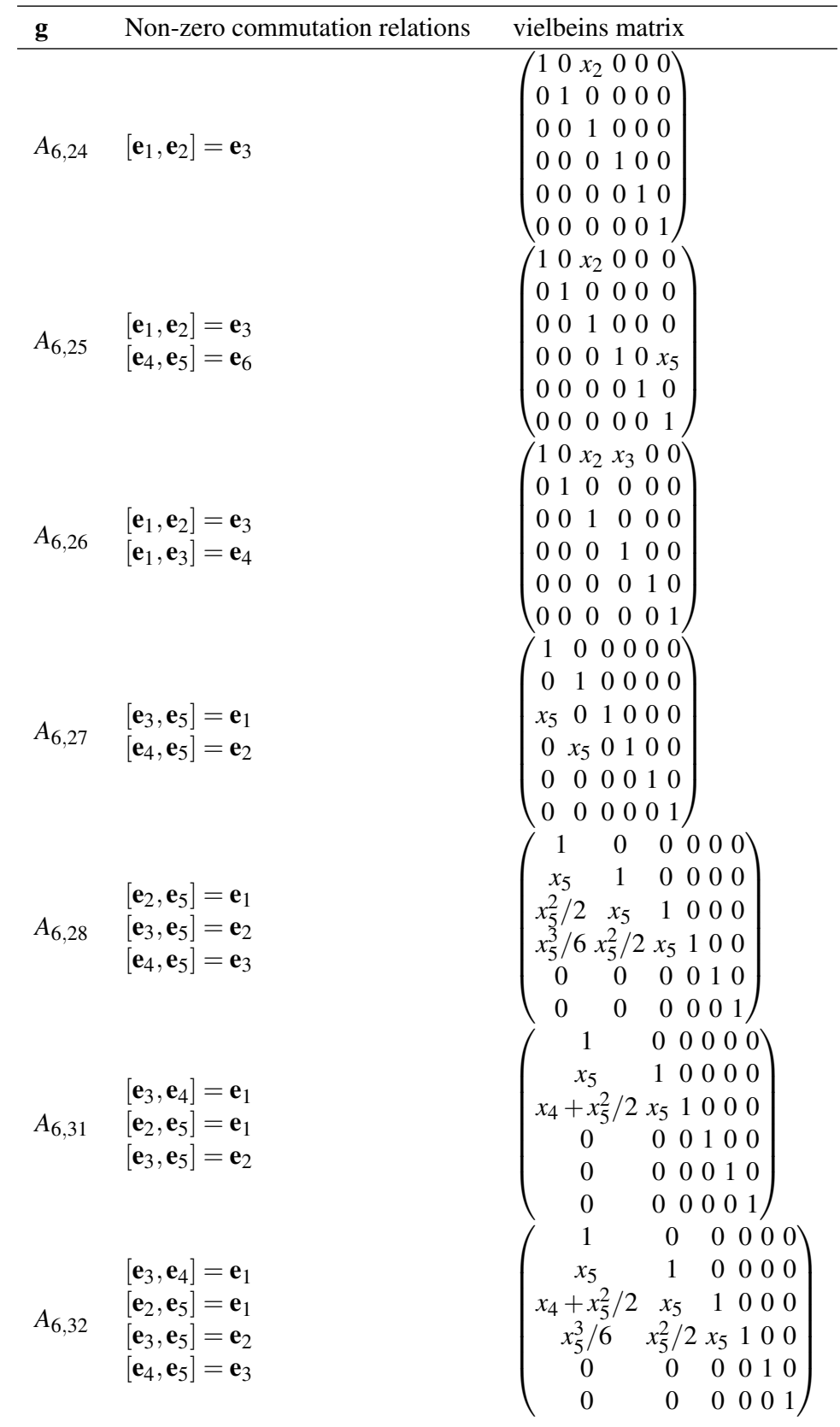

\section{References}

[1] J. Abedi-Fardad, A. Rezaei-Aghdam, and Gh. Haghighatdoost, Classification of four-dimensional real Lie bialgebras of symplectic type and their PoissonLie groups, Theor. Math. Phys. 190:1 (2017) 1-17 (arXiv:1505.04612v1).

[2] A. Bolsinov, Compatible Poisson brackets on Lie algebras and completeness of families of functions in involution, Izv. Akad. Nauk SSSR Ser. Mat. 55 (1991) 68-92. 
[3] A. V. Borisov, I. S. Mamaev and V. V. Sokolov, A New Integrable Case on so(4), , Dokl. Phys. 46 (2001) 888-889; V. V. Sokolov, One Class of Quadratic so(4) Hamiltonians, Dokl. Math. 69 (2004) 108-111; A.V. Tsiganov and O.V Goremykin, Integrable Systems on so(4) Related to XXX Spin Chains with Boundaries, J. Phys. A, 37 (2004) 4843-4849; A.V. Bolsinov and A.A. Oshemkov, Bi-Hamiltonian Structures and Singularities of Integrable Systems, Regul. Chaotic Dyn. 14 (2009) 431-454 ; A.V. Bolsinov, Singularities of Bi-Hamiltonian Systems, Comm. Math. Phys. 331 (2014) 507-543.

[4] R. Campoamor-Stursberg, Symplectic Forms on Six-dimensional Real Solvable Lie Algebras I, Algebra Colloquium. 16 (2009) 253-266.

[5] V. C. Drinfel'd and V. V. Sokolov, Lie algebras and equations of Korteweg-de Vries type, J. Sov. Math. 30 (1985) 1975-2036; Equations of Korteweg-de Vries type and simple Lie algebra, Sov. Math. Dokl. 23 (1981) 457-462.

[6] A. Ibort, F. Magri and G. Marmo, Bihamiltonian Structures and Stackel Separability J. Geom. Phys. 33 (2000) 210-228.

[7] M.A. Jafarizadeh and A. Rezaei-Aghdam, Poisson-Lie T-duality and Bianchi type algebras, Phys. Lett. B 458 (1999) 477-490. (arXiv:hep-th/9903152).

[8] Y. Kosmann-Schwarzbach, F. Magri, Poisson-Nijenhuis structures, Ann. Inst. H. Poincare, 53 (1990) 35-81.

[9] Y. Kosmann-Schwarzbach, F. Magri, Lax-Nijenhuis operators for integrable systems, J. Math. Phys. 37 (1996) 6137-6197.

[10] F. Magri, A simple model of the integrable Hamiltonian equation, J. Math. Phys. 19 (1978) 1156-1162.

[11] F. Magri, P. Casati, G. Falqui, M. Pedroni, Eight lectures on integrable systems, In: Integrability of Nonlinear Systems (Y. Kosmann-Schwarzbach et al. eds.), Lect. Notes Phys, 495 (2nd edition), (2004) 209-250.

[12] F. Magri and C. Morosi, A Geometrical Characterization of Integrable Hamiltonian Systems through the theory of PoissonNijenhuis manifolds, Dipartimento di Matematica F. Enriques, (1984).

[13] B. Mojaveri, A. Rezaei-Aghdam, (4+1)-Dimensional Homogeneous Anisotropic String Cosmological Models, Int. J. Mod. Phys. A. 27, 1250032 (2012).

[14] V. V. Morozov, Classification of nilpotent Lie algebras of sixth order, Izv. Vyssh. Uchebn. Zaved. Mat. 4 (1958) 161-171. (Russian)

[15] M. Nakahara, Geometry, Topology and Physics, Adam Hilger (1991).

[16] G. Ovando, Four dimensional symplectic Lie algebras, Beitr. Algebra Geom. 47 (2006) 419-434.

[17] J. Patera, R. T. Sharp, P. Winternitz and H. Zassenhaus. Invariants of real low dimension Lie algebras, J. Math. Phys. 17 (1976) 986-994.

[18] A. Perelomov, Integrable systems of classical mechanics and Lie algebras, Birkhäuser, (1990).

[19] A. Rezaei-Aghdam, M. Sephid, S. Fallahpour, Automorphism group and ad-invariant metric on all six dimensional solvable real Lie algebras, (arXiv:1009.0816).

[20] A. V. Tsiganov, On bi-integrable natural Hamiltonian systems on the Riemannian manifolds, J. Nonlinear Math. Phys. 18 (2011) 245-268. (arXiv:1006.3914).

[21] A. V. Tsiganov, On bi-Hamiltonian geometry of the Lagrange top, J. Phys. A: Math. Theor. 41, 315212 (2008). 